\title{
The race to the bottom: approaching the ideal glass?
}

\section{Patrick Royall}

HH Wills Physics Laboratory, Tyndall Avenue, Bristol, BS8 1TL, UK.

School of Chemistry, University of Bristol, Cantock Close, Bristol, BS8 1TS, UK. Centre for Nanoscience and Quantum Information, Tyndall Avenue, Bristol, BS8 $1 F D, U K$.

\section{Francesco Turci}

HH Wills Physics Laboratory, Tyndall Avenue, Bristol, BS8 1TL, UK. Centre for Nanoscience and Quantum Information, Tyndall Avenue, Bristol, BS8 $1 F D, U K$.

\section{Soichi Tatsumi}

Kyoto Institute of Technology, Hashiue-cho, Matsugasaki, Kyoto, 606-8585, Japan.

\section{John Russo \\ School of Mathematics, University Walk, Bristol, BS8 1TW, UK.}

\section{Joshua Robinson}

HH Wills Physics Laboratory, Tyndall Avenue, Bristol, BS8 1TL, UK.

\begin{abstract}
Key to resolving the scientific challenge of the glass transition is to understand the origin of the massive increase in viscosity of liquids cooled below their melting temperature (avoiding crystallisation). A number of competing and often mutually exclusive theoretical approaches have been advanced to describe this phenomenon. Some posit a bona fide thermodynamic phase to an "ideal glass", an amorphous state with exceptionally low entropy. Other approaches are built around the concept of the glass transition as a primarily dynamic phenomenon. These fundamentally different interpretations give equally good descriptions of the data available, so it is hard to determine which - if any - is correct. Recently however this situation has begun to change. A consensus has emerged that one powerful means to resolve this longstanding question is to approach the putative thermodynamic transition sufficiently closely, and a number of techniques have emerged to meet this challenge. Here we review the results of some of these new techniques and discuss the implications for the existence - or otherwise - of the thermodynamic transition to an ideal glass.
\end{abstract}




\section{Introduction}

Glass is among the most enduring materials of everyday life, and continues to enjoy new applications, from the covers of our mobile phones [1], core of electrical transformers [2] to advanced non-volatile computer memory [3, 4]. Yet, the dramatic dynamic slowdown encountered upon cooling a glassforming liquid, with its 14 orders of magnitude increase in viscosity/structural relaxation time - that is termed glass transition - continues to elude our understanding. Among the key questions is whether or not this massive increase in viscosity is accompanied by some kind of underlying thermodynamic phase transition [5, 6]. Some theories assume such a transition [7, 8, 9] while others are based on an avoided transition [10] or assume that the glass transition is a predominantly dynamical phenomenon [11. A number of excellent reviews [5, [6, 12, 13, 14] and shorter perspectives [15, 16, 17, 2, 18] of the glass transition from a theoretical viewpoint have appeared recently. More specific reviews focus on certain theoretical aspects such as the energy landscape [8, 19, 20], dynamic heterogeniety [21, 22], Mode-Coupling Theory [23, 24], random first-order theory (RFOT) [9], replica theory [25, 26], dynamic facilitation [11], topological constraints [27] and soft glassy rheology [28] or materials such as polymers [29] or metallic glassformers [30].

If such an underlying phase transition exists, it may be related to the emergence of an "ideal glass", which would reside at the bottom of the free-energy landscape (excluding crystal configurations which represent the true minimum of the landscape). By "ideal glass", we refer to the counterintuitive idea that at sufficiently low (but finite) temperature the entropy of the supercooled liquid falls below the entropy of the crystal [5]. This putative transition temperature is typically determined by extrapolation to low temperatures.

At best, the ideal glass can be approached asymptotically upon cooling a liquid, due to the (presumed) divergence of the relaxation time (and hence the time required to equilibrate the material) $\$$. Recently, considerable developments have been made regarding how close this (putative) state can be reached. Indeed, some experiments are now within $4 \%$ of the temperature at which the ideal glass would be found [31, 32, 33]. Here we review some of these recent developments, both experimental and computational, in techniques to approach the bottom of the energy landscape.

Scope of this work - The intense activity in this field of investigating very deeply supercooled states in a vast range of materials (from metals to molecules to colloids, not to mention many ingenious simulation approaches) means that any kind of comprehensive review is a major challenge. Where possible, we have referenced relevant review papers, but in any case we humbly ask for patience on the part of readers regarding those papers we have missed, or where our opinion seems at odds with theirs. This review is organised into four sections. In the following, section 2, we briefly summarise the state-of-the-art of the field and provide contextual background. We

$\ddagger$ Except for perfectly strong liquids whose relaxation exhibits an Arrhenius dependence on temperature. 


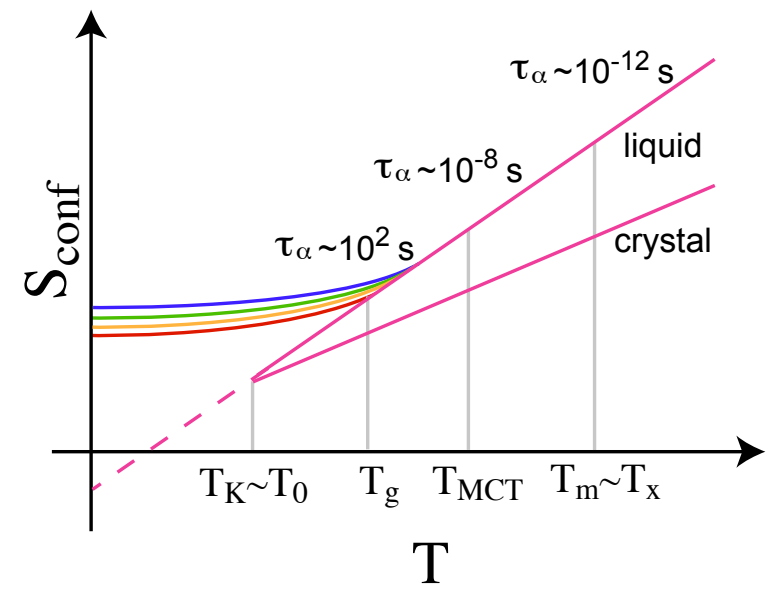

Figure 1. Roadmap to the glass transition. Configurational entropy $S_{\text {conf }}$ as a function of temperature. Typically, the entropy of liquids falls faster than that of crystals as as function of temperature. This suggests that, at some low temperature - the Kauzmann Temperature $T_{K}$ - the liquid entropy would fall below that of the crystal. $T_{K}$ is numerically close to $T_{0}$, the temperature at which the structural relaxation time $\tau_{\alpha}$ is predicted to diverge by the Vogel-Fulcher-Tamman expression Eq. 2 . $T_{g}$ is the operational glass transition temperature where the structural relaxation time reaches $100 \mathrm{~s}$. $T_{\text {mct }}$ is the mode-coupling transition, $T_{m}$ is the melting point and $T_{x}$ denotes a crossover temperature below which relaxation occurs through cooperative motion, i.e. the energy landscape becomes significant.

then review recent developments in experiments in section 3 before turning to computer simulations in section 4. We summarise and conclude our discussion in section 5 .

\section{Background : paradigm for the ideal glass}

What is the glass transition? - The nature of the glass transition, if any, is not understood. On timescales accessible to experiment and computer simulation, the glass transition takes the form of a continuous increase in viscosity, or structural relaxation time. In normal liquids, the relaxation occurs on the picosecond timescale. When the relaxation time reaches $100 \mathrm{~s}$, a supercooled liquid is termed a glass. This is a purely operational definition (it is easier to time $100 \mathrm{~s}$ than a divergent timescale!) which can obscure the fact that at the temperature corresponding to a relaxation time of $100 \mathrm{~s}$, $T_{g}$, precisely nothing happens in a thermodynamic sense, so $T_{g}$ does not correspond to any kind of true glass transition. Put another way, $T_{g}$ could as well correspond to a relaxation time of $10 \mathrm{~s}$ or $1000 \mathrm{~s}$. Thus whether we have a supercooled liquid or a glass depends, not necessarily on the material or state point, but upon how long we wait.

That the structural relaxation time grows to unmanageable timescales has significant consequences. The relaxation time is an indicative measure of how long it takes a liquid to reach equilibrium. At least 10-100 relaxation times are required before one can say that a liquid is "in equilibrium". As the relaxation time reaches 100s, a 


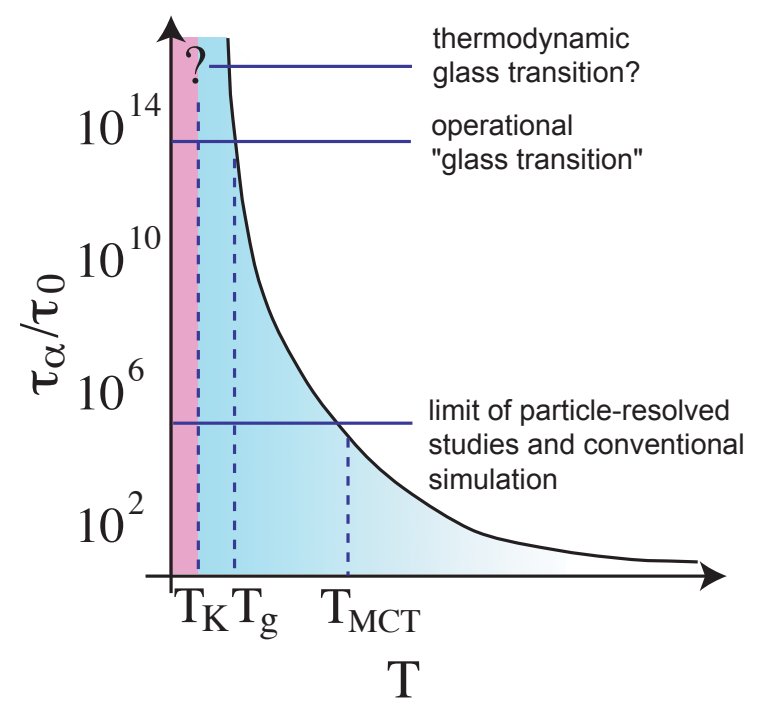

Figure 2. The challenge of the glass transition. The operational glass transition, $T_{g}$ is the limit of most experiments, some 14 decades in relaxation time slower than the normal liquid. This still doesn't reach the putative ideal glass transition at the Kauzmann temperature $T_{K}$. Conventional computer simulations and particle-resolved colloid experiments are hampered even further, as they are limited to only around five decades.

material will appear solid. It will no longer flow on reasonable timescales. This gradual emergence of solidity that characterises the glass transition can obscure a fundamental question. Because it is very hard to equilibrate the material below $T_{g}$, it is very hard to answer questions about whether there is a true thermodynamic transition to an ideal glass at some lower temperature $T_{K}<T_{g}$. It is this equilibration issue that we seek to address here.

The ideal glass — It is suggested in some theoretical approaches [5, 7, 9, 26] that there is a thermodynamic transition at or around the Kauzmann temperature $T_{K}$ where upon extrapolation, the entropy of the supercooled liquid falls below that of the crystal [34]. We emphasise that it is quite possible that this paradox, of an amorphous state having a lower (or equal) entropy than the crystal, may be avoided, for example by crystallisation [34, 35] or by other effects, such as thermal defects [36]. Indeed, arguments have been made against the existence of the so-called Kauzmann paradox [11, 37, 38]. In particular, it is important to re-iterate the point made in the preceding section: any such ideal glass is not expected to represent the true minimum of the energy landscape, as that would correspond to the crystal [37]. Other possibilities that the ideal glass might be avoided include thermal defects [36], or that it is not even expected in theories such as dynamic facilitation [11. However, a number of theoretical approaches, Adam-Gibbs and RFOT amongst them [7, 9] invoke a drop in the configurational entropy in the amorphous state as a mechanisms for vitrification. In this way of thinking, the system is presumed not to undergo crystallisation and thus the true bottom of the energy landscape, corresponding to the crystal, is neglected. 
Rather than comparing the entropy with respect to the crystal, as Kauzmann did in his original 1948 paper [34], in much of the work covered in this review, we are interested in the entropy in the amorphous state only. For our present purposes, we note that the supposed existence of this Kauzmann point, existent or otherwise, nevertheless serves as a useful landmark when considering the behaviour of glassforming systems $[2,5,6,8,19,12,13,14,15,16,17,18,19,20,25,26]$, and we therefore use the Kauzmann point to guide our approach to deeply supercooled states low in the energy landscape.

Often, the approach to the bottom of the energy landscape is thought of in terms of the configurational entropy of the supercooled liquid, which is expected to become very small [7, 9]. For our purposes, the configurational entropy $S_{\text {conf }}$ is defined as $S_{\text {conf }}=S-S_{\text {vib }}$, where $S$ is the total entropy of the system, and $S_{\text {vib }}$ is the vibrational entropy due to thermal excitations around inherent states, i.e. the energy minima that are reached following a steepest descent quench. Note that the vibrational entropy $S_{\text {vib }}$ also implicitly includes contributions from the entropy of mixing in the case of multicomponent systems [39]. $T_{K}$ is defined as the temperature at which $S_{\text {conf }}$ becomes sub-extensive, meaning that it vanishes in the thermodynamic limit. The Kauzmann paradox observes that the liquid entropy falls faster than that of the crystal and at a finite temperature $T_{K}<T_{g}$, the two should cross [34].

Here we focus on the configurational entropy, $S_{\text {conf }}$ (Fig. 1). While we follow the ideas of Ref. [39], we emphasise that this splitting of the entropy into vibrational and configurational parts is valid provided there is a sufficient decoupling between vibrational timescales ( $\beta$-relaxation) and timescales of full relaxation ( $\alpha$-relaxation). This implies a certain degree of supercooling [39]. However, this may be operationally challenging [40], and recent computer simulations have explored different ways to determine the configurational entropy (see Section 4.2] [41] depending on the precise definition of the vibrational contribution. Given that the configurational entropy of the crystal is small, this suggests that the ideal glass should have an exceptionally low configurational entropy while nevertheless remaining amorphous (Fig. 1). Alternatively, there may be no transition until absolute zero temperature [6, 5, 11]. The focus of this work is to review recent progress towards addressing this conundrum.

Another significant point is the mode-coupling temperature at $T_{\text {mct }}$ where, according to this particular mean-field theory, a divergence of the relaxation time is predicted to occur [23]. In its usual implementation, mode-coupling theory does not include the effect of activated processes so its associated dynamical divergence is never truly observed $\S$. But echoes of this mean field transition can be observed in experiment, and it also marks the emergence of activated relaxation.

In this discussion, we have identified three relevant temperatures : $T_{g}$ the experimental transition, $T_{K}$ the possible thermodynamic transition to the ideal glass,

$\S$ Extensions to MCT which have the potential to include collective relaxation include inhomogeneous MCT [42, Generalised MCT [43] and hopping, which enables a link to be made between MCT and random first-order transition theory, thus connecting MCT with theories which make a direct link to the energy landscape [44, 45]. 
and $T_{\text {mct }}$ the mode-coupling temperature. An important point is that in considering dynamical arrest, one should think of glass transition-s, or that at least we must specify what we mean by "glass transition". Here we focus on the approach to $T_{K}$. We now briefly review some key concepts concerning the behaviour of systems as they move low in their free energy landscape. We note that recently, a further transition in amorphous systems has been identified. This Gardner transition occurs between two glass states, but is not pertinent to our discussion of the quest for the ideal glass [25, 46].

\subsection{Fragility and the Angell Plot}

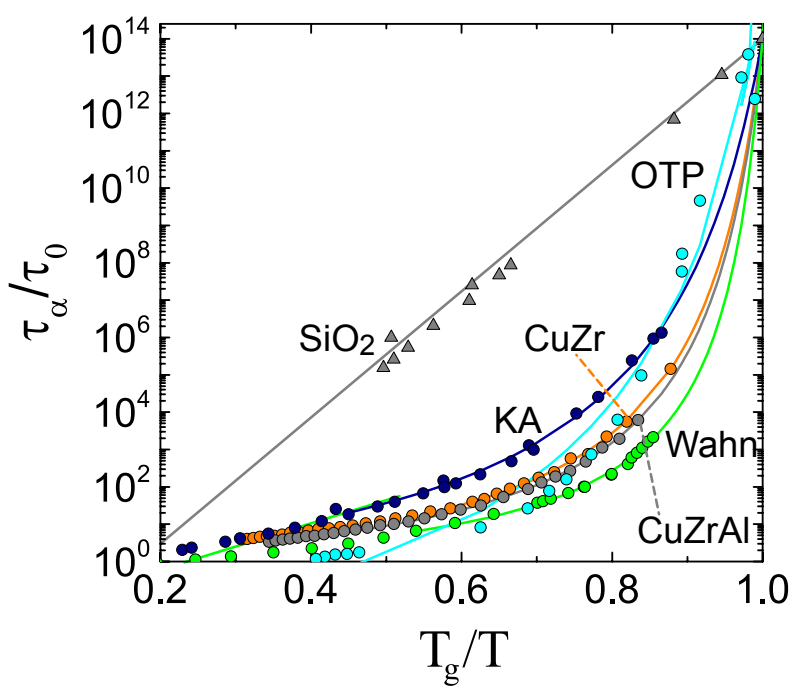

Figure 3. The Angell plot : Arrhenius representation of liquid viscosities, with inverse temperature scaled by $T_{\mathrm{g}}$. Strong liquids exhibit Arrhenius behaviour characterised by an approximately straight line, indicative of a temperature-independent activation energy. Fragile liquids, on the contrary, reveal super-Arrhenius behaviour, where activation energy grows as temperature decreases [47]. Data for $\mathrm{SiO}_{2}$ and orthoterphenyl (OTP) are quoted from Angell [48] and Berthier and Witten [49]. The other data concern model systems: KA denotes Kob-Andersen and Wahn denotes Wahnström binary Lennard-Jones systems respectively while HS denotes hard spheres where the control parameter is the compressibility factor $Z$ [50, 51]. CuZr and $\mathrm{CuZrAl}$ denote numerical calculations of metallic glassformers by ourselves based on an embedded atom model for $\mathrm{Cu}_{65} \mathrm{Zr}_{27.5} \mathrm{Al}_{7.5}$ [52. The latter matches the composition of the experiments by $\mathrm{Yu}$ et al. [53]. Here, $\tau_{0}$ is scaled to enable data collapse at $T_{g} / T=\phi / \phi_{g}[54$.

The Angell plot [47] offers a convenient representation of the dynamical slowing down underlying the glass transition, and highlights some of the most important differences between glassforming materials. In this plot, the logarithm of the structural relaxation time $\tau_{\alpha}$ (or the viscosity) is plotted against $T_{g} / T$. In this representation, an Arrhenius-like behaviour,

$$
\tau_{\alpha} \propto \exp \left(\frac{E_{\mathrm{a}}}{k_{\mathrm{B}} T}\right)
$$


where $E_{\mathrm{a}}$ is viewed as an activation energy, should be represented by a straight line. In Fig. 3, we plot the logarithm of the viscosity, which (neglecting Stokes-Einstein breakdown [5]) is proportional to $\tau_{\alpha}$, showing that for some materials, notably silica, it is indeed close to a straight line. However, for many supercooled liquids a fragile or super-Arrhenius behavior is found. The increase in relaxation times is often well described by the semi-empirical Vogel-Fulcher-Tamman (VFT) expression,

$$
\tau_{\alpha}=\tau_{0} \exp \left[\frac{D T_{0}}{T-T_{0}}\right]
$$

where $T_{0}\left(\simeq T_{\mathrm{K}}\right)$ is rather lower than the experimental glass transition temperature $T_{\mathrm{g}}$ and $D$ is a measure of the fragility - the degree to which the relaxation time increases as $T_{g}$ is approached. Smaller values of $D$ correspond to more fragile materials. Furthermore, Eq. 2 can be rationalised by both Adam-Gibbs [7] and random-first order transition [9] theories. Remarkably, $T_{0} \approx T_{K}$ for a large number of glassformers, fuelling the idea of a thermodynamic phase transition at $T_{K}$ \|. For our purposes, let us emphasise that for a large number of materials, $T_{0}$ and $T_{K}$ vary from one another by $10 \%$. While it is worth noting (see earlier, Section 2 ) that the accurate determination of $T_{K}\left(\right.$ and $\left.T_{0}\right)$ is not without its challenges, more fundamentally, one expects a significant connection with fragility. It is suggested that more fragile materials exhibit more change in structure, and thus their configurational entropy should also change more [55] (see also section 2.2). Conversely, less fragile (i.e. stronger) materials exhibit a smaller change in structure, and indeed are rather poorly fitted by the VFT expression Eq. 2, so it comes as no surprise that strong materials such as silica exhibits differences in $T_{K}$ and $T_{0}$ of $15 \%$ [56. Some polymers, notably polycarbonate and polyamide exhibit more substantial deviations (the latter has a $T_{0}$ over $30 \%$ above its $T_{K}$ ) [57]. Nevertheless, very many glassformers do have $T_{0}$ and $T_{K}$ close together.

As successful as Eq. 2 has been (at least for fragile glassformers), it is not the only option. In fact, as Tab1 indicates, the value of $T_{K}$ deviates from $T_{0}$. Dyre and coworkers showed that VFT is not the best fit to experimental data [58, and other forms, for example one also related to the Adam-Gibbs theory [59], provide better agreement with experimental data. Furthermore, the Elmatad-Garrahan-Chandler form [60], based on dynamic facilitation theory [11] fits the experimental data just as well, and assumes no dynamic divergence at finite temperature. Other examples in which no dynamic divergence is encountered include expressions such as that developed by Mauro et al. [59] which relate configurational entropy the topological degrees of freedom per atom, elastic treatments of excitations which enable relaxation [61, geometric frustration [10] and related models which treat glassformers with a population dynamics model of locally favoured structures [62. Nevertheless, here we shall use the VFT form and presume that the divergence at $T_{0}$ is representative of $T_{K}$ [6], for ease of comparison with earlier work [63].

\| See Cavagna [6] for an interpretation of $T_{0} \approx T_{K}$ 


\subsection{Dynamic and static lengthscales}

Dynamic Heterogeneity - Approaching the glass transition, dynamic heterogeneity becomes a significant feature: Particles move in an increasingly cooperative manner creating dynamically correlated mesoscopic domains [64, 65]. A dynamic length scale can be associated with the increasing dynamic heterogeneity - a measure which characterizes the size of growing cooperative motion. This lengthscale can be quantified by using so-called four-point correlation functions. The fluctuations associated with dynamic heterogeneity are then characterised by the dynamic susceptibility,

$$
\chi_{4}(t)=\frac{1}{N \rho A_{\mathrm{B}} T}\left[\left\langle W^{2}(t)\right\rangle-\langle W(t)\rangle^{2}\right],
$$

which is obtained by integrating a four-point time-dependent density correlation function over volume [66]. For a given temperature $T$ (or packing fraction $\phi$ ), the fluctuations (i.e., the susceptibility) attain a maximum at certain time $t=\tau_{h}\left(\approx \tau_{\alpha}\right)$, and then die away, as illustrated in Fig. 4. The time where $\chi_{4}(t)$ is maximised $\tau_{h}$ has a value similar to $\tau_{\alpha}$.

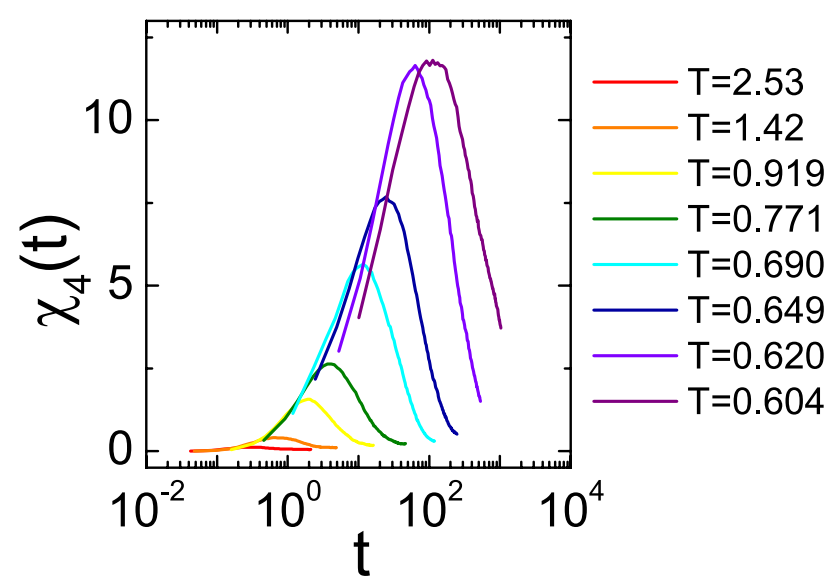

Figure 4. The dynamic susceptibility $\chi_{4}(t)$ in the Wahnström binary Lennard-Jones glassformer. Colours correspond to different temperatures 67 .

The exact nature of the increase of the dynamic length scale remains unclear, and depends on the system under consideration. In simulations of the Wahnström binary Lennard-Jones glassformer, this increase of a dynamic lengthscale was likened to divergence of the static density-density correlation length observed in liquid-gas critical phenomena [66]. However it has also been claimed that the increase of the dynamic lengthscale related to $\chi_{4}, \xi_{4}$ supports the dynamic facilitation interpretation (divergence of the dynamics only at $T=0$ ) [68], while other work points to a divergence at the VFT temperature $T_{0}$ [69]. Recent work involving deep supercooling (by the standards of computer simulation) and careful analysis indicates a change in scaling of $\xi_{4}$ around the mode-coupling crossover [70]. Such a dynamic lengthscale cannot be measured directly in experiments on molecules. However indirect measurements have been made 
in a variety of ways, all arriving at similar conclusions: the size of the cooperatively rearranging regions (CRRs) is only around 10-100 molecules at $T_{g}$, where the dynamics are ten decades slower than around the mode coupling temperature, close to the limit of supercooling that can be reached via simulations [22, 33, 171, 72, 173, 74, 75, 76, 777. We are thus left with the unsettling situation that simulations and experiments obtain similar values for the dynamic length when the dynamics differ by ten decades. These studies underline the difficulties of obtaining sufficiently supercooled samples where the lengthscale changes enough to enable a clear discrimination to be made.

The rationale for a static lengthscale - Building on ideas introduced by Bouchard and Biroli, [78], Montanari and Semerjian considered a cavity made of immobile particles outside a certain radius, and where re-arrangements are possible inside [79]. Now, above a certain cavity size, particle re-arrangements are just possible, and thus $\tau_{\alpha}$ is finite. In other words, there is a lengthscale, above which there is more than one way to arrange the particles in the cavity. Under these conditions, the system must be able to relax and a maximum relaxation time $\tau_{\alpha}^{\max }$, which corresponds to the transition between these different configurations. This cavity has a fixed lengthscale, and, under these assumptions, $\tau_{\alpha}^{\max }$ should be Arrhenius, as we have that there are multiple configurations inside the cavity and there is some activation barrier — whose height fixes $\tau_{\alpha}^{\max }$, i.e. there is some fixed maximum activation energy $E_{a}^{\max }$ in Eq. 1. Of course, especially in the liquid at temperature $T$ above the melting temperature $T_{m}$, the system relaxes much faster than $\tau_{\alpha}^{\max }$. However, if $\tau_{\alpha}$ follows a super-Arrhenius behaviour, it must, eventually, cross $\tau_{\alpha}^{\max }(T)$. This would mean that the assumption of a cavity with the number of available configurations constant with respect to temperature is flawed. In other words, under super-Arrhenius dynamics some structural change must occur. i.e. the system moves lower in its energy landscape.

In addition to the arguments above, theories which imagine a thermodynamic origin to the glass transition [7, 9, 10, 26] predict that static lengthscales should increase concurrently with dynamic lengthscales. In the majority of investigations (limited to the weak supercooling regime to $T_{\text {mct }}$ accessible to computer simulations), the dynamic $\left(\xi_{4}\right)$ and static lengths appear decoupled [67, 80, 81, 82, 83, 84, 85, 86, 87, 88, 89, 54, 90, 91], with the possible exception of weakly polydisperse hard-spheres [69, 92, 93, 94] and and certain innovative measures of local solidity [95]. It is possible that other dynamic lengths might resolve this issue [90], but in our opinion, there is a need to obtain data at deeper supercooling than has been possible hitherto. The role of lengthscales in the glass transition has recently been reviewed by Karmakar et. al [96] and Harrowell [97].

\subsection{The Energy Landscape}

One very intuitive picture of the equilibrium dynamics of a deeply supercooled liquid is provided by Goldstein's energy landscape [5, 8. Goldstein put the emphasis on the evolution of the system in phase space, i.e. the space of all the configurational degrees of freedom. In the case of a simple liquid in three dimensions, this is the space of all $3 \mathrm{~N}$ 

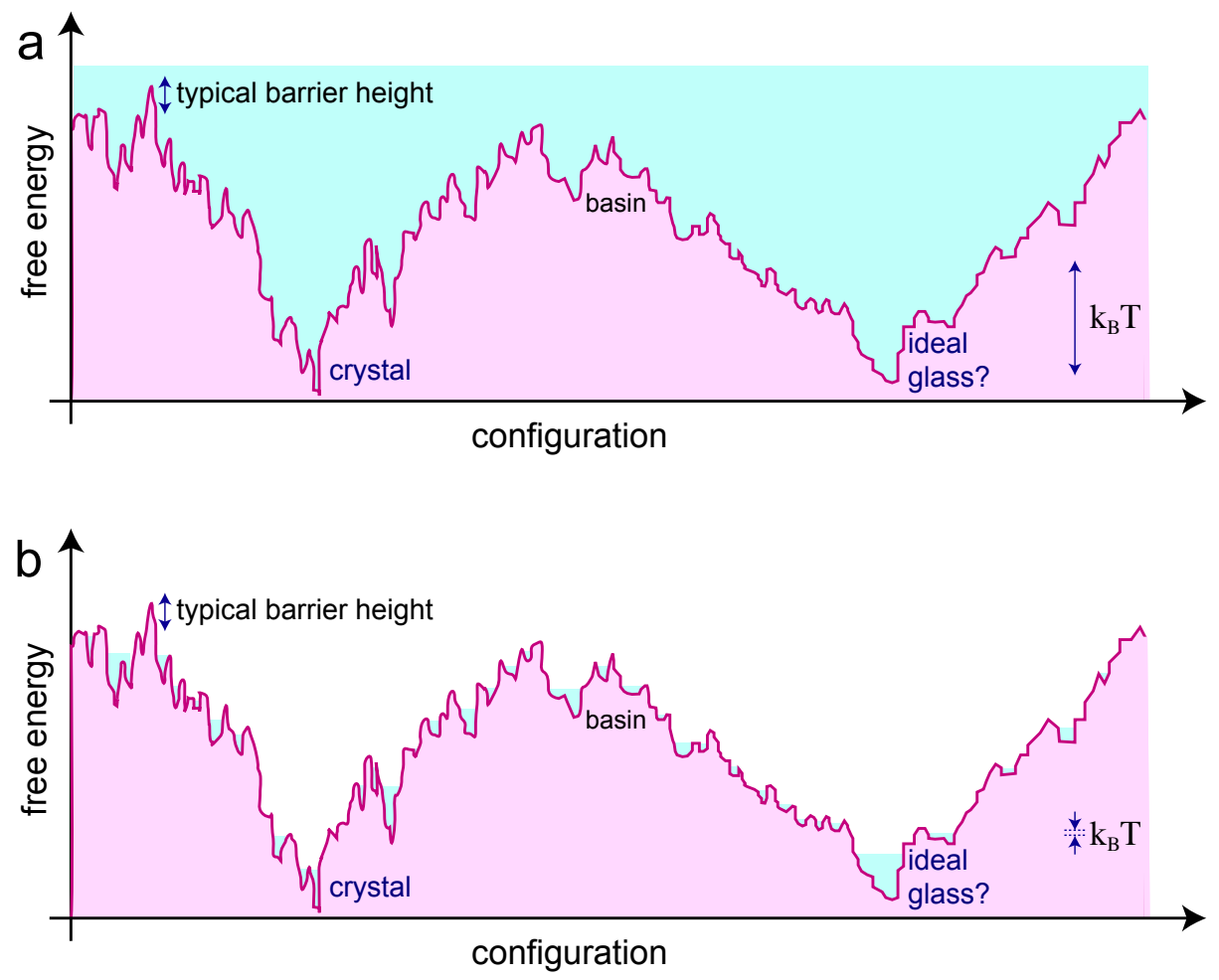

Figure 5. A schematic illustration of the potential energy landscape. The $x$-axis represents configurations of all $3 N$ coordinates. The bottom of the energy landscape is typically the crystal, but the energy minimum for the ideal glass may be comparable. (a) High temperature liquid, the typical barrier height is less than the thermal energy, and all configurations can be accessed as indicated in blue. (b) Low temperature glass. The barrier height between basins is now much higher than $k_{B} T$, and the system is no longer able to explore all configurations and has become a glass but can only access local states, as indicated by the blue shading.

coordinates of the particles. The total potential energy is then $U\left(r_{0}, r_{1} . . r_{N}\right)$ where the $r_{i}$ are the coordinates of the $N$ particles which comprise the system. Each configuration is represented by a point in phase space, and the dynamics of the system can be thought of as the motion of this point over this potential energy landscape. In other words, such a classical system is entirely described by its energy landscape [6]. The local minima of the potential energy correspond to mechanically stable configurations of the particle system. One of these is the crystal, and this will usually be the absolute minimum (Fig. 5). Beyond the crystal-related minima, there will be many local minima corresponding to particle arrangements that lack crystalline order. These are amorphous, or glassy, minima, and have a potential energy that is larger than the crystal one.

Below some crossover temperature ( $T_{x}$ in Fig. 11), a supercooled liquid explores the phase space mainly through activated hops between different amorphous minima, which are separated by potential energy barriers. Note that it is in (meta) equilibrium (metastable to crystallisation), so the potential energy is constant in time, save for 
thermal fluctuations (we assume there is no ageing) and the system is ergodic [6]. One should note that these hopping events involve only a few particles (the typical number of dynamically correlated particles can be estimated by $\chi_{4}(t)$, Eq. 3. Fig. 4).

Henceforth we review recent developments in approaching the bottom of the energy landscape (neglecting crystallisation). We begin by considering experimental approaches, and move on to consider computer simulations.

\section{Approaching the bottom of the energy landscape in experiments}

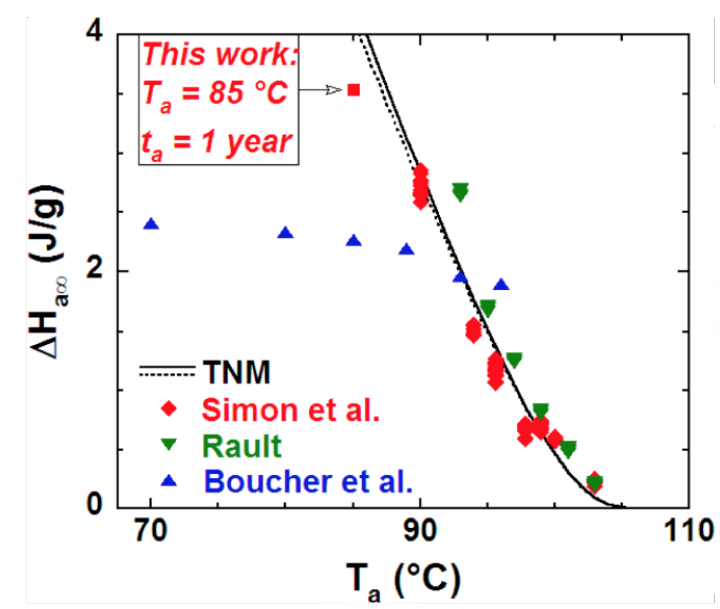

Figure 6. Aging experiments on polystyrene glass. Enthalpy loss at equilibrium $\left(\Delta H_{a, \infty}\right)$ as a function of temperature. Koh and Simon's work is shown as the red squares. The black solid line is a theoretical prediction from Tool-NarayanaswarmyMoynihan 198, 99, 100, which takes into account the temperature dependence of change in heat capacity $\Delta C_{p}$, and the black dotted line is the prediction with fixed value of $\Delta C_{p}$ at $T_{g}$. The data point from this work is a lower bound. The key point of this figure is that the $\Delta C_{p}$ of Koh and Simon's work corresponds to that of an equilibrium supercooled liquid $4 \%$ below $T_{g}$ in absolute terms. Reprinted with permission from Y. P. Koh and S. L. Simon. Macromolecules, 46 5815-5821 (2013). Copyright 2013 American Chemical Society.

Here we review strategies adopted in experiment which can obtain states in the $T_{K}<T<T_{g}$ regime. Our intention is to address the question of how low in the energy landscape experiments have reached. Most of our analysis will consider molecular systems, as these sample phase space very much faster than their colloidal counterparts. The latter struggle even to pass the mode-coupling transition, and remain many orders of magnitude in terms of relaxation time from $T_{g}$ in the definition for molecules, that the relaxation time should be 14 decades more than that of the liquid, $\tau_{\alpha}\left(T_{g}\right) / \tau_{\alpha}\left(T>T_{m}\right) \sim 10^{14}$.

To assess how close to the bottom of the energy landscape the system has reached, we shall employ the criteria introduced by Ediger and coworkers 63 .

$$
\theta_{0}\left(T_{\mathrm{eq}}\right)=\frac{T_{g}-T_{\mathrm{eq}}}{T_{g}-T_{0}}
$$




\begin{tabular}{|c|c|c|c|c|c|c|c|c|}
\hline system & $T_{K}, Z_{K}$ & $T_{0}, Z_{0}$ & $T_{g}, Z_{g}$ & $T_{\text {eq }}, Z_{\text {eq }}$ & $D$ & $\theta_{0}$ & $m$ & Technique \\
\hline EB & 10133 & $95.5[101]$ & 1116 & 10532 & 8.30 & 0.53 & 97.5101 & VD 32 \\
\hline $\mathrm{ECH}$ & 76102 & 67.1103 & 101 & 94104 & 21.5 & 0.19 & 56.5105 & VD 102. 104 \\
\hline TNB & 270106 & 264107 & 345 & 30663 & 14.1 & 0.48 & 86 107] & $\mathrm{VD} 63$ \\
\hline $\mathrm{TL}$ & 10833 & 98.2108 & 117 & 108 109] & 7.51 & 0.48 & 104 101] & VD 109] \\
\hline IMC & 240110 & 234111 & 314 & 28663 & & 0.36 & $82.8 \overrightarrow{111}$ & $\mathrm{VD} 63$ \\
\hline PR & 49.833 & & 5633 & & & & & $\mathrm{VD} 33$ \\
\hline $\mathrm{CCl}_{4}$ & & 69 112 & $78.5 \overrightarrow{112}$ & & 4.54 & & 118112 & $\mathrm{VD} 112$ \\
\hline $\mathrm{MG}$ & & 625113 & 67653 & 64053 & 2.85113 & 0.70 & 3853 & $\mathrm{VD} 53$ \\
\hline PS & & 321 114 & 373 & 361 115 & 6.17 & 0.23 & 116114 & $t_{\mathrm{w}} \sim 1$ year 115 \\
\hline PCB & 290116 & 374114 & 424 & 408117 & 3.37 & 0.31 & 91.6114 & $t_{\mathrm{w}} \sim 1$ year 117 \\
\hline $\mathrm{AMB}$ & & 338 [118 & 409 & 366 119] & 7.29 & 0.61 & 87 [118] & $t_{\mathrm{w}} \sim 2 \times 10^{7}$ year 119 \\
\hline OTP & $225[120$ & 202121 & 246 & 23038 & $\sim 10[121$ & 0.36338 & $81[121$ & $t_{\mathrm{w}} \sim 4$ months 38 \\
\hline $\mathrm{SiO}_{2}$ & * & & $820-900$ & & $\sim 60$ & & 20122 & \\
\hline PHS & 45.45 & & 33 & 38 & & 0.40 & $\approx 50$ & Particle swap MC [123. 41] \\
\hline HS & 28.8 & 27.0 & & $\dagger$ & 69.74 & & & MD, colloid exp. 124, 54, 51] \\
\hline $\mathrm{KA}$ & 0.325 & $0.357(5)$ & & $\dagger$ & $3.62(8)$ & & & MD 85, 54, 125. 126] \\
\hline Wahn & $0.464(7)$ & $0.488(5)$ & & $\dagger$ & $1.59(13)$ & & & MD 67, 54] \\
\hline
\end{tabular}

Table 1. League table for the bottom of the energy landscape. The equilibrated temperatures $T_{\text {eq }}$ in this table are collected from the literature specified in each row [63, 109, 119] or estimated by using enthalpy diagrams [32, 104, 117] or estimated the upper limit of $T_{\text {eq }}[53$ by using the relation between deposition temperature and $T_{\text {eq }}$ specified in ref. [127. EB is ethylbenzene, ECH is ethylcyclohexane, TNB is 1,3-bis-(1-naphthyl)-5-(2-naphthyl)benzene, TL is toluene, IMC is indomethacin, PR is propene, $\mathrm{CCl}_{4}$ is carbon tetrachloride, $\mathrm{MG}$ is the metallic glass, $\mathrm{Cu}_{65} \mathrm{Zr}_{27.5} \mathrm{Al}_{7.5}$. PS is polystyrene, PCB is polycarbonate, AMB is amber, OTP is ortho-terphenyl, $\mathrm{SiO}_{2}$ is silica, PHS (very) polydisperse hard spheres, HS hard spheres, KA denotes the Kob-Andersen mixture, Wahn the Wahnström mixture and $t_{w}$ is the waiting time. VD denotes vapor deposition, MD denotes conventional molecular dynamics simulations. Note that, as a strong liquid, the fragility of silica is poorly defined [48].

$\dagger$ No simulations except the particle swaps [41, 123, 128, have yet been equilibrated at deeper supercooling than the experimental glass transition $T_{g}$.

where $T_{\text {eq }}$ is the temperature at which the system has been equilibrated. Originally $T_{K}$ is used to represent the ideal glass. However, a value for $T_{K}$ is often harder to obtain than $T_{0}$. Thus to discuss a wider range of systems from the literature, we recall that $T_{0} \approx T_{K}$ for many systems [6]. As a result, we consider the extent of traversing between $T_{g}\left(\theta_{0}\left(T_{g}\right)=0\right)$ and $T_{0}\left(\theta_{0}\left(T_{0}\right)=1\right)$.

\subsection{Annealing}

Implicit in Fig. 1 is that simply waiting longer than $100 \mathrm{~s}$ and reducing the temperature in a commensurate fashion will enable a material to equilibrate at temperatures below $T_{g}$. In this way, it is conceptually straightforward to equilibrate a material below $T_{g}$, however the key question is how far below $T_{g}$ one can reach in a "reasonable" waiting time. Inspection of Fig. 3 indicates that for fragile glassformers, waiting times quickly become very long indeed before the material can be equilibrated. If we take one year as our "reasonable" waiting time, then the work of Koh and Simon [115, who looked at the enthalpy of polystyrene, suggests that with a year of aging one can try to equilibrate 
to temperatures of $12 \mathrm{~K}$ below $T_{\mathrm{g}}$ or $3 \%$ in absolute terms (see table 1 ). This means the system can reach $11 \%$ above $T_{0}$, or $\theta_{0}=0.23$ in Eq. 4. Other examples inlcude the work of Aliotta et al [38], who obtained a fictive temperature of $230 \mathrm{~K}$ for orthoTerphenyl (OTP). This is consistent with the model of Tool [98], Narayanaswarmy [99] and Moynihan 100] (TNM).

The TNM model is based on the assumption that relaxation can be attributed to a fictive temperature. Here the term fictive temperature refers to the temperature that an equilibrated material would have, were it to exhibit the same behaviour as the aging material, as determined by some observable, such as the relaxation time. In this case, the fictive temperature is greater than the true temperature of the material, so the relaxation time relevant to the annealing process becomes much faster than the prediction from extrapolation of the VFT expression (Eq. 2), which would pertain to the fully aged, equilibrium material. Significantly, the assumption of the TNM model implies the existence of a fast relaxation component. While other materials with differing fragilities may offer closer approaches to $T_{0}$ with this conceptually straightforward method, the prediction from the TNM model suggests that without massive increases in waiting time, an approach to around $10 \%$ of $T_{\mathrm{K}}$ is to be expected.

One way around the limitations of annealing is to use thin films, of polymeric glassformers in particular [129]. One recent and exciting result is that it has been shown that thin films of can exhibit fictive temperatures which approach their Kauzmann temperature, and that further annealing does not [130].

Before moving on to other strategies to approach the bottom of the energy landscape, we note here an intriguing recent work, which identifies two relaxation timescales in metallic glasses just below their glass transition $T_{g}$ [131]. The additional fast mode of relaxation was suggested by the authors to relate to stress-driven relaxation. It is in fact possible that such behaviour may relate to spinodal gelation [132, 133, 134]. Certainly, model glassforming systems are routinely quenched quite close to their gasliquid demixing line [135, 136].

\subsection{Waiting for a long time: Amber}

Another strategy to attain the bottom of the energy landscape is to let nature do the waiting, and have the experiment start some time ago. This approach has been pursued by McKenna and coworkers, who studied fossilized amber, a glass forming biopolymer, which had been aged for 20 million years [119]. For amber, ambient temperature (taken as $300 \mathrm{~K}$ ), is around $100 \mathrm{~K}$ below its $T_{g}(409 \mathrm{~K})$ and about $40 \mathrm{~K}$ below its $T_{0}(338$ $\mathrm{K})$. As shown in Table 1, the vast timescale of millions of years has taken the fictive temperature to within $8 \%$ of $T_{0}$, the same degree with aging experiments just below $T_{g}$ in the laboratory for one year [115, although application of Eq. 4 shows a more promising $\theta_{0}=0.61$. It is worth noting here that McKenna and coworkers inferred that the dynamics of amber below $T_{g}$ should be Arrhenius, which implies that there is no Kauzmann ideal glass transition at all : the material simply becomes gradually 
slower until absolute zero is reached [119]. However, this analysis presumes a single characteristic relaxation time. In other polymers [137] and metallic glasses [131], multiple relaxation times have been observed upon recovery following aging, suggesting a potentially more complex situation.

\subsection{Ultrastable Glasses}

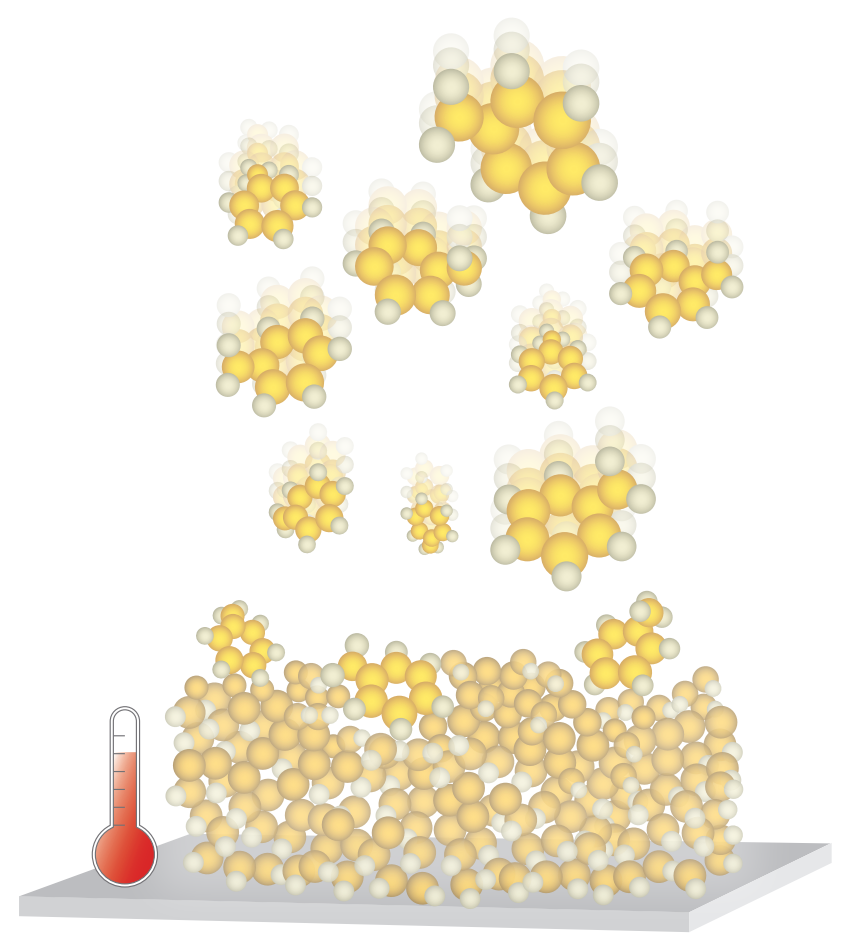

Figure 7. Reaching for the bottom of the energy landscape using free surfaces. Vapordepositing glass forming molecules where the substrate is at around $0.85-0.93 T_{g}$ leads to ultrastable glasses [32, 104, 63, 109, 112, 53. Molecular mobility near the free surface is believed to be the key to producing a such ultrastable glasses. The incoming molecules can reach equilibrium if the substrate is close to $T_{g}$. Reproduced from [15].

In a landmark paper [63], Ediger and coworkers demonstrated a new and very powerful means to access extreme states towards the bottom of the energy landscape. The method to produce these ultrastable glasses is to deposit e.g, indomethicin (IMC) or 1,3-bis-(1-naphthyl)-5-(2-naphthyl)benzene (TNB) molecules, one-by-one from a vapor onto a substrate as indicated in Fig. 7. The depth to which the material can reach in the energy landscape is quantified via its fictive temperature, which is determined from calorimetry and density measurements. The value of the substrate which minimises the fictive temperature is around $0.85 T_{g}$. Due not least to their novel material properties, the field of ultrastable glasses has seen remarkably rapid growth, for which we refer the reader to the very recent review by Ediger [2]. 
Subsequent work suggests this technique can lead to conditions which exceed $\theta_{0}=0.5$, or that the material is equilibrated to within $10 \%$ of $T_{0}$ [31, 32]. Excepting perhaps the intriguing work of McKenna and coworkers (see above) [138, 139], this represents the closest approach yet achieved in experiment to $T_{\mathrm{K}}$. However in the case of a number of molecular glassfomers [140, 141, there is evidence of anisotropy in the thin films of many ultrastable glasses [142, 141]. In a sense, when comparing to conventional liquid-cooled glasses, this suggests that there is some fundamental change in the nature of the material. Nevertheless, the potential of the vapor deposition technique is further illustrated by its applicability to a diverse range of molecules [2, 102, 104, 109, 112] some of which, such as toluene [143], are more isotropic in shape and even to metallic glassformers, in which the interactions are roughly spherical [53]. From the work done on the metallic glassformers by Yu et al. [53], we estimate an astonishing approach to the ideal glass, in the sense that the parameter $\theta_{0}=0.7$ or within $7 \%$ of $T_{0}$. The equilibration temperature $T_{\text {eq }}$ of the system is estimated from extrapolation of the enthalpy in the same manner with other vapor-deposited glass shown in other work [32, 63]. We note that we could not find an estimation of $T_{0}$ (or $T_{K}$ ) in the literature so we employed numerical simulation with an embedded atom model on the same system as shown in Fig. 3. We thus estimate $T_{0}$ as $626 \mathrm{~K}$.

The key to forming these ultrastable glasses appears to lie in the mobility of the surface molecules. Stevenson and Wolynes showed that simple arguments based on Random First Order Transition Theory predict a value of $\theta_{0}=1 / 2$, due to a halving in the free energy barrier to relaxation in the presence of a free surface. In practise the vapour deposition by which the ultrastable glasses are formed allows for some further equilibration, i.e. it appears to be possible to equilibrate to lower temperatures, $\theta_{0}>1 / 2$ [144. These free surface dynamics have been probed in experiment. Using a novel technique specifically designed to probe surface relaxation, by using a tobacco mosaic virus placed on the surface, and using atomic force microscopy to probe the mobility, Fahkraai and coworkers have shown that surface dynamics are decoupled from the bulk and follow an Arrhenius-like scaling [145, 146]. This Arrhenius scaling may enable a system to get even deeper in the energy landscape than has been possible thus far.

Ultrastable glasses correspond to materials with relaxation times, in the conventional sense, that extend to spectacular timescales. One may nevertheless enquire as to whether there might be some way to deduce a relaxation time, and indeed such an approach has been pursued by investigating the sound velocity in such materials. The results are shown in Fig. 8. Remarkably, these point to an Arrhenius behaviour for the relaxation time - i.e. no dynamical divergence until absolute zero and thus no transition to an ideal glass [147]. Interestingly, these results are consistent with those of Mckenna and coworkers who studied hyper-aged amber (section 3.2).

One final point to note about ultrastable glasses, suggesting that they may be genuinely novel states, is their tunnelling properties. Quantum tunnelling is typically encountered, and rationalised by a two-state model in conventional liquid-cooled glasses and may be related to small voids in the glass. Ultrastable glasses, on the other hand, 


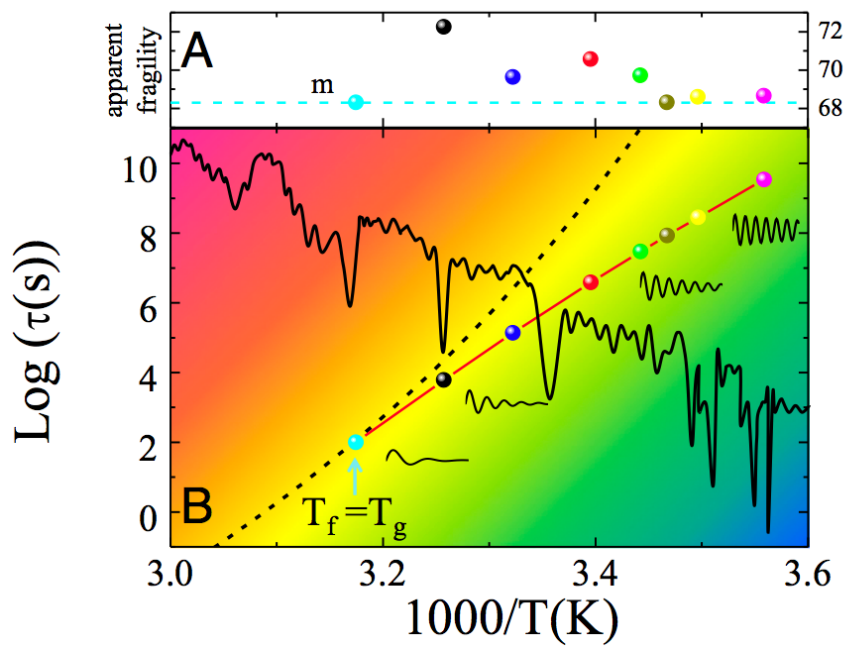

Figure 8. Viscous flow of a supercooled liquid in the exapoise range. (a) Apparent fragility determined from the sound velocity jump in glasses with different fictive temperatures indicates a fragile to strong transition below $\mathrm{T}_{g}$. Conventional fragility $m=$ is also reported. (b) Arrhenius plot for $T<T_{g}$, obtained by integrating data from (a). A correlation is found between the depth of the inherent structures in the energy landscape (gray line) probed by glasses of different fictive temperature $T_{f}$ and the mechanical response (sketched by time-domain damped oscillations). The VFT behavior obtained from above $T_{g}$ data is also shown (dashed line). Reproduced from [147.

do not appear to exhibit such two-state tunnelling [148]. Other glassformers which access deep into the energy landscape, such as fossilised Amber, do exhibit tunnelling [149]. It is worth noting that hyperquenched glasses (quenched while still quite high in the energy landscape) exhibit enhanced low-frequency relaxations relative to annealed systems [150].

\subsection{Hypothesis on ultrastable glasses and their relation to fragility}

As we saw in the previous section, physical vapour deposition at an appropriate temperature allows us to make ultrastable glass even for metallic glassformers, whose constituent atoms interact in an isotropic fashion, as compared to molecular glassformers, which is supported in computer simulation of systems with spherically symmetric interactions (see section 4.2). This suggests that the ability of materials to form ultrastable glasses may be rather general, and so here we provide a discussion concerning relaxation in thin films and provide a connection to fragility.

The process of the realization of the vapor-deposited ultrastable glass is pictured as follows; Firstly, highly energized molecules, i.e. vapor, attach to the surface whose temperature is somewhat lower than $T_{g}$. Second, only a few molecules on the surface are energized and mobile, which over time relax to a local energy minimum and cease moving (apart from local vibrations).

We noted above in section 2.2 that high fragility implies a larger energy barrier to 


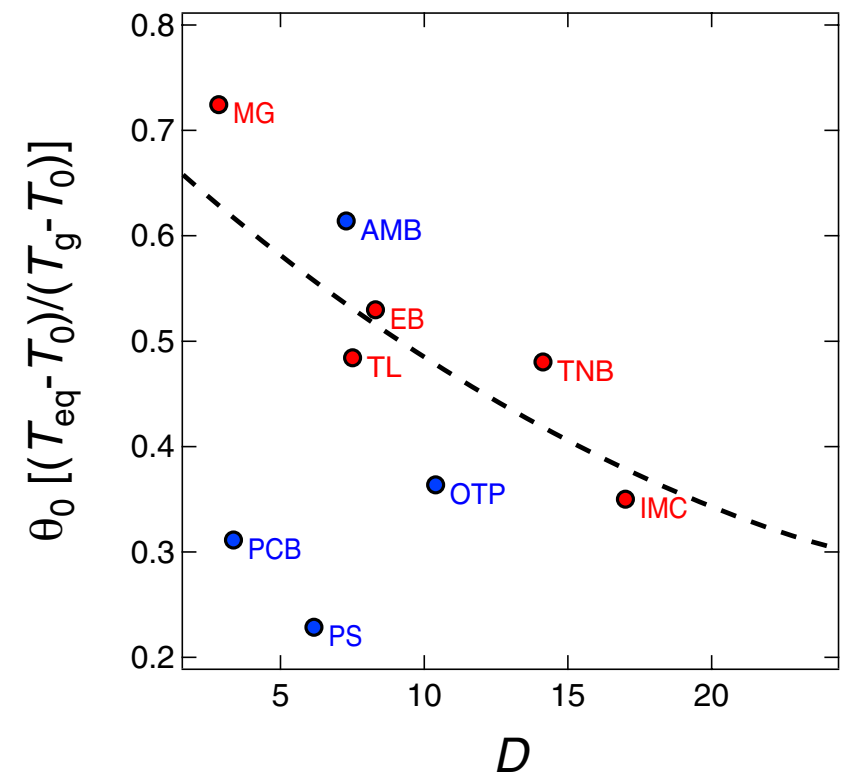

Figure 9. Approaching the bottom of the energy landscape: the role of fragility. Here we plot $\theta_{0}$ vs fragility parameter $D$. The dashed line is a guide to the eyes. Remarkably, a negative correlation is found between $\theta_{0}$ and fragility parameter $D$ across various substances. Red circles indicate data sets obtained by physical vapour deposition and blue circles indicate data sets obtained by annealing. Abbreviations shown to the right of each plots are the same as in Tab. 1

relaxation and that, within the framework of for example Adam Gibbs [7] or random first-order transition theories [9], this would be related to a decrease in configurational entropy and thus the cooperatively rearranging regions would increase in size. What does this mean for thin films? We suppose that the influence of the surface in enabling relaxation in the film is limited to a lengthscale beyond which it has little effect. Let us further suppose that this surface lengthscale is related to the CRR size. Therefore, if more fragile materials indeed have longer co-operative lengthscales, the size of cooperatively rearranging regions might grow sufficiently that the ability to relax via the surface, crucial for ultrastable glasses, would be lost. Conversely, for strong glassformers, with small co-operative lengthscales, the ability of the surface to enable relaxation may be retained at much deeper supercooling. How might this conjecture be tested? The degree of supercooling made possible by the vapour deposition is characterised by Eq. 4 . We therefore plot $\theta_{0}$ as a function of fragility, characterised by the parameter $D$ in Eq. 2 in Fig. 9. Now $D$ measures the inverse fragiity, so we expect a negative correlation, which is precisely what we find for a wide range of materials. Note that in Fig. 9, we also plot annealed materials (blue data points), whose scatter is very much larger. This lack of correlation in bulk (non-vapour-deposited) systems supports the idea of a link between fragility and the effectiveness of vapour deposition in approaching the bottom of the energy landscape. 


\subsection{Experiments on Colloids}

Before closing the discussion on experimental approaches to access the bottom of the energy landscape, we briefly consider colloids. These systems follow the same rules of equilibrium statistical mechanics as do atoms and molecules, and are sought-after for four properties. Firstly, they can be directly imaged. In particle-resolved studies [151, 152, 153], their dynamics are tracked at the single-particle level yielding data otherwise available only to simulation [154, 151, 155, 156]. Secondly, relative to molecules their large size leads to sluggish dynamics, so time-resolved data can be taken [157, 158] but this means that it is hard to access the kind of timescales associated with the molecular glass transition with colloids (Figs. 1 and 3). Therefore, for the purposes of probing the depths of the energy landscape, colloids cannot hope to compete with molecules. Thirdly, the interactions between the colloidal particles can be tuned, and only classical interactions need be considered. This means that they form sought-after model systems against which theories based on, for example hard spheres, can be readily compared. Finally, and crucially for our discussion, colloids can be manipulated, in particular by the use of optical tweezers [159, 160, 161, 162].

The sluggish dynamics of colloids leads to a common — and important misconception. This is that the relaxation time at the experimental glass transition $T_{g}$ is somehow absolute. Molecules are said to vitrify when the relaxation time exceeds $100 \mathrm{~s}$, which is an entirely anthropocentric quantity. This is around 14 orders of magnitude longer than relaxation timescales in high-temperature liquids. On the other hand colloids can have relaxation times of $\sim 100 \mathrm{~s}$ without exhibiting any traces of slow dynamics. This obviously presents a major problem: If we employ for colloids the same criteria as those applied to molecular fluids, we find that the "molecular" $100 \mathrm{~s}$ corresponds to $\sim 10^{8}$ years. In other words, in order to presently complete the equivalent dynamic range of 14 orders of magnitude in particle-resolved techniques on colloids, the measurement would need to have commenced in the Jurassic period! (and while this is possible with experiments on amber (section 3.2), the particle-resolved experiments are in practise limited to a few days duration [163]). In other words, dinosaurs made little use of particle-resolved studies of colloids.

In terms of colloids reaching for the bottom of the (free) energy landscape, the most deeply supercooled experiments have just passed the mode-coupling transition [164]. As modest as this is in comparison with molecules, the strength of the colloids lies in the quality of information delivered: no other experimental technique delivers $3 \mathrm{~d}$ timeresolved particle coordinates comparable to computer simulation. In the future we may hope to go more deeply supercooled than has been achieved so far, but even without passing $T_{g}$ it should nonetheless be possible to discriminate some of the competing theoretical approaches, due to the richness of the data obtained. Furthermore, the degree of manipulation possible means that some techniques from computer simulation may be employed in colloidal systems. One the one hand, these experiments serve as experimental verification of theoretical approaches, and on the other hand, they show 


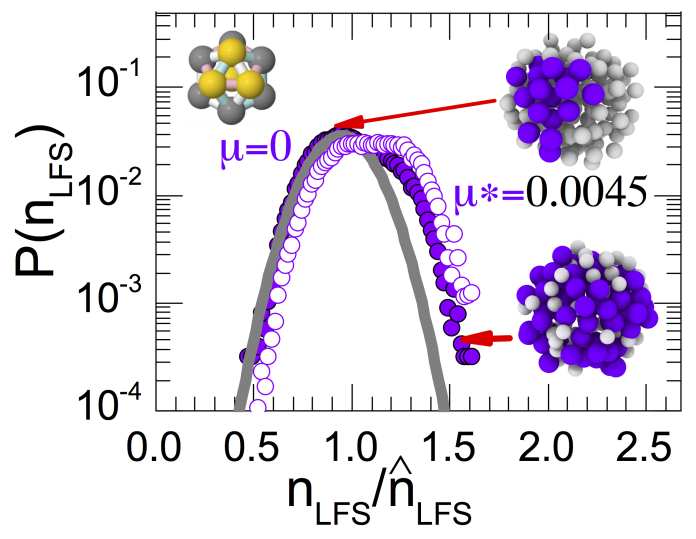

Figure 10. Probability distributions of populations (filled symbols) of particles in locally favoured structures in trajectories sampled from experimental data. The locally favoured structure is the defective icosahedron [51, indicated top left. Data is then reweighted according to a dynamical chemical potential $\mu$ (open symbols) to reveal a phase transition at $\mu^{*}$ between a local-structure-rich state (right) and a normal liquid. In the snapshots of the experimental coordinates, purple particles are in defective icosahedra, rendered for each state. The population of purple LFS particles is much higher in the LFS-rich state, indicating that it lies deep in the energy landscape. Reproduced from [169.

that colloidal systems can indeed approach the bottom of the energy landscape more effectively than might be expected from dynamical considerations alone. Considerable progress in this direction has been made through the use of optical tweezers to "pin" particles [165, 161, 160, 162], or spontaneous adsorption in 2d systems [166] (see simulation work in Section 4.3. Meanwhile insight can be gleaned through ingenious experiments on "vapour-deposited" colloidal glasses [167].

Even with current technology, it is possible to approach the bottom of the energy landscape more closely that the arguments above suggest. Using methods developed in computer simulation [168] (see section 4.5), Pinchaipat et al. [169] were able to identify a non-equilibrium phase transition. States identified in this non-equilibrium phase transition correspond to configurations very deep in the energy landscape. Under the assumption that the number of locally favoured structures (geometric motifs associated with supercooling [170]) represents the degree of supercooling, the configuration illustrated in Fig. 10 is at an effective volume fraction $\phi_{\text {eff }}=0.59$, beyond that accessible to conventional techniques. While still not as deeply supercooled as the molecular glass transition, nevertheless this corresponds to a relaxation time around 100 times higher than the typical limit of particle-resolved colloid experiments.

\section{Approaching the bottom of the energy landscape in silico}

Computer simulations of deeply supercooled liquids encounter difficulties analogous to those that afflict experiments: if both the structure and the dynamics of the liquid are to be directly sampled, at low temperatures (or very high packing fractions in 
the case of hard-sphere-like systems) the very slow glassy dynamics makes brute force calculations extremely demanding in terms of computational resources. Around the (avoided) dynamical transition temperature $T_{\text {mct }}$ predicted by Mode Coupling Theory, such brute force calculations rapidly become impossible even for the simplest models (such as polydisperse mixtures of hard spheres or binary mixtures of Lennard-Jones particles) in the sense that (metastable) equilibrium is never attained. The liquid slowly and continuously drifts to lower (free)-energies, undergoing ageing on the accessible simulation timescales.

However, an important advantage of theoretical and numerical approaches with respect to experiments is that it is possible to perform controlled approximations that allow us to circumvent the contingent limits of our computational power. Such approximations rely on a limited number of assumptions, and aim at sampling equilibrium properties, artificially accelerating the local relaxation processes by different means.

Here we summarise some of the most popular and intriguing ideas that have been developed in the last decades to explore deeply supercooled, low (free) energy states: we will first discuss evaluating the energy landscape itself, and then consider highly optimised, non-local Monte-Carlo schemes allowing to rapidly sample the equilibrium configurations of deeply supercooled liquids (event-chain algorithm; particle swapping techniques); we will then consider the route to very large static correlations obtained through particle pinning, inspired by Random First Order Transition theory (RFOT); then, the numerical equivalent of the experimental vapour-deposition techniques will be illustrated, followed by a discussion of trajectory-based techniques that (together with non-equilibrium information) allow us to access very low energy states from the statistics of rare events; we conclude with direct methods of local minimisation and sampling of the potential energy surface. Before exploring the what may loosely be termed the "smart algorithm approach", i.e. the techniques such as those mentioned above, we note that with the advent of code optimised for graphic processing units (GPU), it has now become possible to run brute force simulations for considerably longer than has been possible until very recently: for example, with GPU-codes highly optimised for systems with a few thousands of particles, such as RUMD [171], or with massively parallel codes such as LAMMPS [172, 173] or HOOMD-blue [174, 175], which can scale to thousands of GPUs. Nevertheless, in terms of the approach to the bottom of the energy landscape, even such GPU optimised code appears to be outperformed by some highly specialised algorithms.

\subsection{Evaluating the energy landscape}

In recent years, improvements in computational processing power have enabled development of techniques to investigate some of the predictions of the energy landscape concept [8, 19]. Among the first developments [176, 177] was to establish the inherent structure. The inherent structure is the result of a steepest-descent quench to $T=0$, 
which thus takes the system to its nearest local minimum in the potential energy landscape (Fig. 5). Stillinger and Weber [176, 177] further showed that for low temperatures (where the system is confined to a particular basin for long periods), the entropy could be expressed as a sum of a configurational and vibrational part, $S=S_{\text {conf }}+S_{\text {vib }}$. The vibrational part can be treated harmonically, and combined with the inherent state contribution $S_{\text {conf }}$, it is possible to "numerically evaluate the energy landscape" [20, 39, 178, 179, 180, 181].

Such investigations have largely confirmed Goldstein's energy landscape picture, that the system can be viewed as residing in basins for times of order of the structural relaxation time before undergoing rapid transitions between basins in the energy landscape [8]. In particular, Doliwa and Heuer [182] have investigated local structure in the basins (via the potential energy). Basins with high activation energy barriers for escape corresponded to low potential energy. They found that escape from a particular basin corresponds to a complex multistep process involving a succession of energy barriers. Interestingly from the point of view of local structure, some work has suggested that such transitions involve small compact clusters (which might correspond to co-operatively re-arranging regions) [183], unlike the string-like motion identified in earlier studies [181, 184].

Wales and coworkers have developed powerful numerical techniques to enable the energy landscape of systems with a few hundred particles to be explicitly calculated [185]. This is sufficient to explore glassy behaviour, such that the energy of a representative set of basins in the energy landscape can be expressed in a "disconnectivity graph" (Fig. 11] [186, 187]. Such analysis yields the minimum energy structure, which, in the case of the Kob-Andersen system appears to be crystalline 188. with a striking structural similarity to the bicapped square antiprisms which are the locally favoured structure for this model [189, 85].

\subsection{Non-local Monte-Carlo techniques}

Sufficiently supercooled liquids are characterised by the slow motion of particles, often caged by their neighbours, in a process that dramatically reduces the diffusivity. This phenomenology is well reproduced by thermalised Molecular Dynamics (MD) or local Monte Carlo (MC) schemes, akin to over-damped dynamics [190]. However, caging prevents full equilibration at very low temperatures. It is therefore desirable to construct dynamics different from the real dynamics that can, however, attain the correct equilibrium distribution of states. Several alternative schemes are based on extended ensemble techniques [191], such as replica exchange Markov chain Monte-Carlo (also known as parallel tempering) [192, 193, 194, 195] eventually combined with importance sampling methods such as umbrella sampling [196, 197]. In these methods, the dynamics is modified in the sense that several copies of a glassy system run in parallel and exchange information regularly, so that tunnelling across metastable states is possible in order to obtain well equilibrated samples at low temperatures. In these schemes, the microscopic 


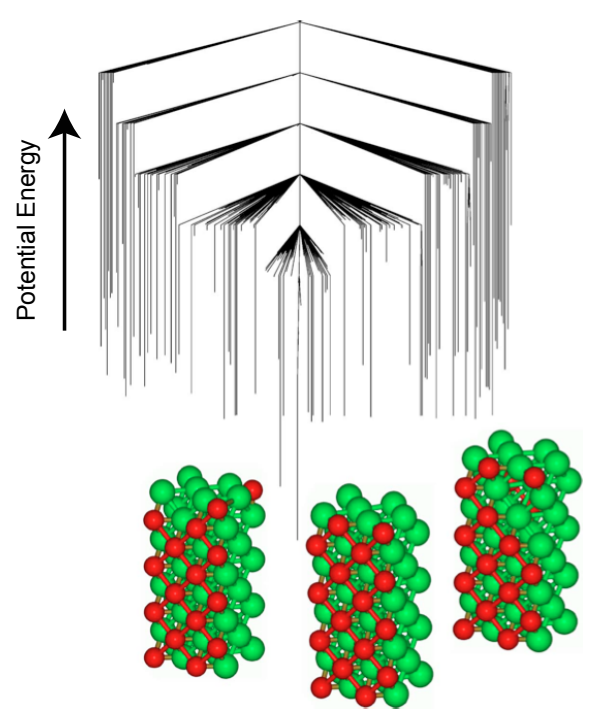

Figure 11. The energy landscape of a $N=320$ Binary Lennard-Jones (Kob-Andersen) system represented as a disconnectivity graph. Three low-lying minima which have a crystal-like appearence are rendered [186, 188. Note the similarity of these crystals to the bicapped square antiprism which is known to be locally favoured structure for this system [189, 85]. Reproduced with permission from American Institute of Physics Copyright 2007.

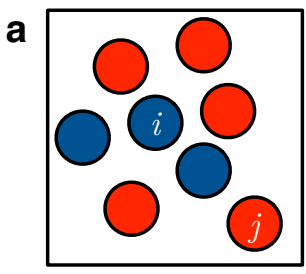

$\mathrm{t}$

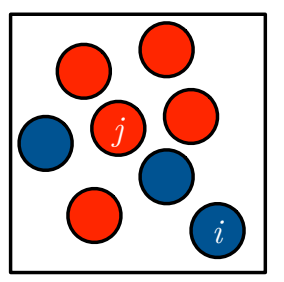

$t+\Delta t$

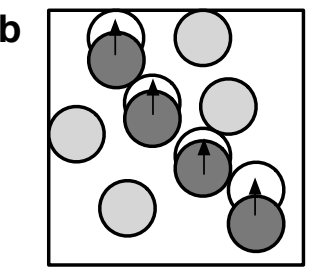

$\mathrm{t}$

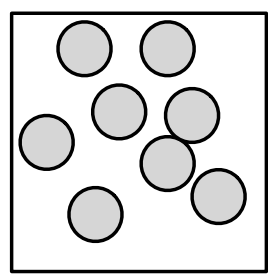

$\mathrm{t}+4 \Delta \mathrm{t}$

Figure 12. Non-local Monte-Carlo schemes. (a) Particle swap in binary mixture of red and blue particles. [123, 128] (b) Straight event-chain Monte-Carlo move: a random direction (in this case along the vertical direction) is chosen and a chain of moves is triggered starting from the particles in the bottom left and involving four particles (dark shading) [198, 199].

dynamics typically remains physical (for example Newtonian or Langevin dynamics) and glassy phenomena such as caging are still preserved, causing the unavoiidable emergence of dynamical slowing down at low temperatures.

One can overcome the issue of caging in a more drastic way if non-local moves of the particles are introduced. These allow faster equilibration time, at the cost of loosing the information on the real dynamics of the system. Particularly successful techniques include the following methods.

Particle Swaps Monte-Carlo Model glassformers are typically mixtures of multiple (at least two) components of different sizes (and sometimes masses). It is possible 
to exploit the difference in species extending the Metropolis-Monte-Carlo algorithm with moves in a space of particle identifiers, stochastically accepting/rejecting swaps of the particle types [200] [see Fig. 12(a)]. This allows for a new kind of particle move, which contributes to a much more rapid exploration of the configurational space. In practice, while in local Monte-Carlo schemes cage-breaks are a cooperative process, particle swaps make them non-cooperative. Particle swapping techniques have been shown to outperform methods such as parallel tempering for soft binary mixtures [201], and very polydisperse mixtures of hard spheres [123]. In general, the efficiency of the method depends on the specific interactions and the composition of the liquids under consideration. In the case of binary mixtures, for example, the efficiency of the method can be affected by undesirable crystallization events (additive mixtures with not too large size ratios) or by very large rejection probabilities (non-additive mixtures such as the Kob-Andersen mixture) [83, 128]. A careful choice of the size distribution (for example, using hybrid mixtures with two main components and a residual component with a broad distribution of possible sizes) allows one to obtain equilibration times that are several orders of magnitude faster than conventional (local) Monte-Carlo methods [128.

Such an acceleration of the dynamics allows us to explore a much broader range of temperatures and access equilibrated states with very low configurational entropy at finite (reduced) pressure, corresponding to an ideal glass transition [41], while in 2d such a transition would appear to be found only at zero temperature [202].

Assuming that these methods sample the same configuration space as conventional techniques, these novel results provide ground for a revived debate on the role of multibody correlations in the emergence of slow dynamics. If, on the one hand, we have clear evidence for the growth of static correlations and the reduction of configurational entropy (regardless of its specific definition, as demonstrated in [41], see Fig. 13), on the other hand the simple fact that swap methods push the dramatic slowing down to much lower temperatures suggests that the observed dynamic arrest in real systems (which certainly cannot undergo swap moves) is mostly due to the slowdown of local dynamics [203], although this suggestion has been challenged with the idea of crumbling metastability [204], and a mode-coupling theory based approach similarly leads to dynamical arrest which is suppressed but essentially unaltered particle swaps [205]. Nevertheless, the idea of a dominant local dynamics of Wyart and Cates [203] appears to be corroborated by recent mean-field replica liquid [206] and effective potential [207] calculations that account for the effect of swap dynamics, suggesting that for the extreme case of such polydisperse systems the slowing down would not be related to the actual metastable states but to kinetic effects.

The particle swaps enable a powerful tool to test ideas which have been previously inaccessible. Examples include demonstrated that surface mobility is indeed the mechanism for the deeply supercooled ultrastable glasses (see section 3.3] 208] and provide evidence in support of the non-equliibrium Gardner transition between glasses and jammed-like states [46, 209]. 


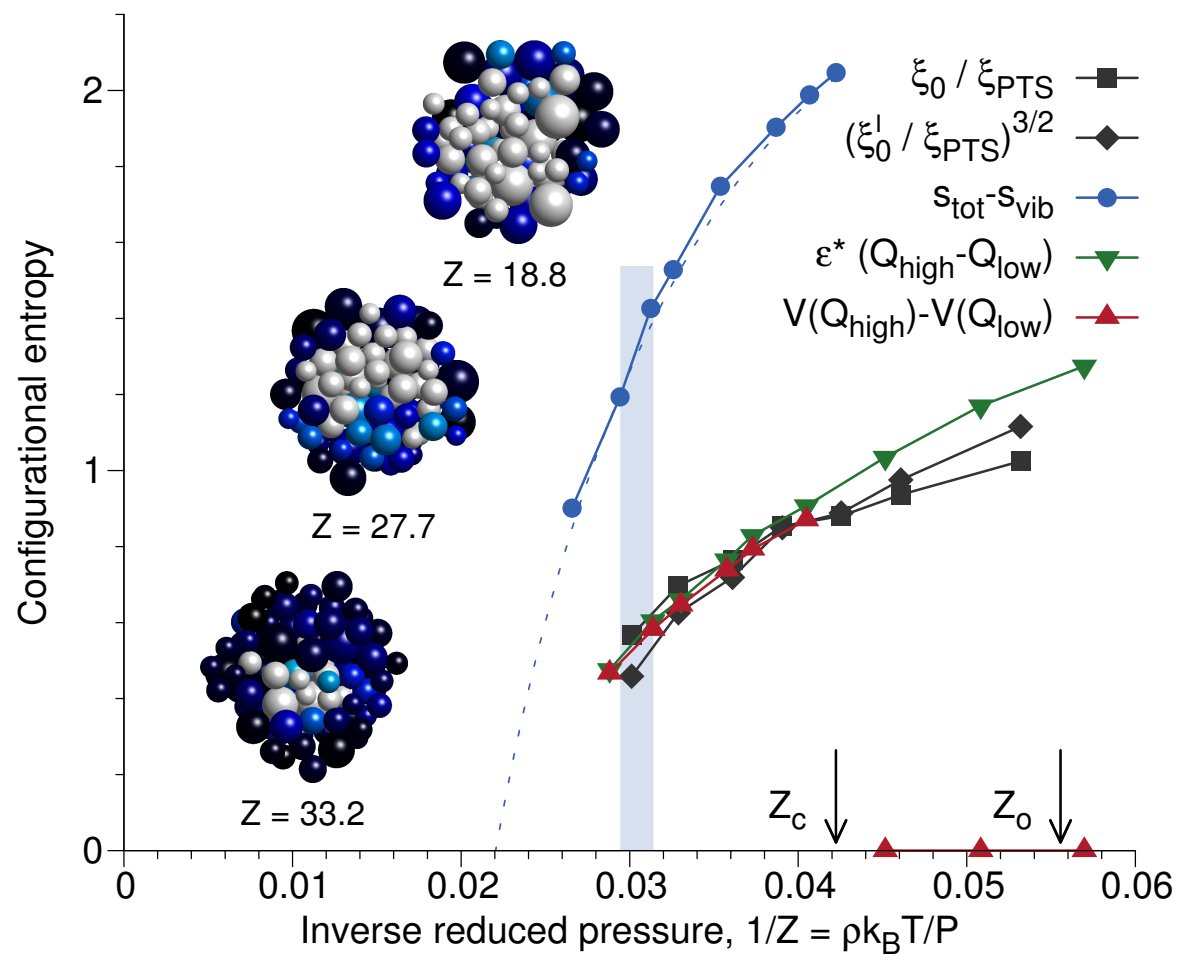

Figure 13. Configurational entropy in a system of polydisperse hard spheres measured according to several indicators (scaled point-to-set length $\xi_{\text {PTS }}$, configurational entropy from thermodynamic integration $S$ tot $-S_{\text {vib }}$, from differences in the Franz-Parisi potential on overlap fluctuations $V\left(Q_{\text {high }}\right)-V\left(Q_{\text {low }}\right)$, from the susceptibility in biased simulations $\left.\varepsilon^{*}\left(Q_{\text {high }}-Q_{\text {low }}\right)\right)$. The inset illustrates overlap profiles in cavities. Reproduced from [4].

Event-Chain Monte-Carlo An alternative route consists of implementing Monte-Carlo schemes that accelerate the equilibration through long-range coherent motions of the particles [198, 199]. Such so-called event-chain Monte-Carlo algorithms efficiently sample equilibrium configurations minimising [210] or avoiding [199] rejections, and are therefore particularly suitable for dense systems. In fact, event-chain algorithms have been used to successfully assess the first-order nature of the transition from liquid to hexatic order in two-dimensional hard disks [198], and recently applied to binary mixtures of hard disks [211, 212].

In its simplest form (for example, hard-core interactions), the method consists in a deterministic chain of events occurring in randomly distributed directions: a random particle and a random direction are initially selected; the particle moves till it hits a second particle, which moves in the same direction till it hits a third particle, and so on until an arbitrary total displacement is performed [see Fig. 12(b)]. Such moves are repeated in random directions and ensure that only valid configurations are sampled. The method can be adapted to break detailed balance [213] while still preserving the correct probability measure [199] (leading to a further performance boost) and also to work with continuous interaction potentials [214, 215, 216]. 


\subsection{Pinned particle techniques}

At very low temperatures, the mobility of the particles of a super-cooled liquid is much reduced, with a highly heterogeneous spatial distribution. The size of the least mobile regions increases as the temperature is reduced, and a so-called dynamical length scale, increasing by a factor of $5-10$, is associated with this process in many different glass forming systems [22, 67, 217, 218, 219, 220, 97, 96, 221, 222]. The definition of an analogous static length scale, growing as the system is cooled, has proven to be challenging [90, 170, 189], due to the modest impact that the supercooling has on immediate measures of static correlations such as the radial distribution functions, and to observe a well-coupled growth of structural and dynamic lengthscales one needs to go to the limit of weak geometric frustration [223], not suitable for good glass formers. Higher order correlation functions have therefore been developed and so-called pointto-set (PTS) correlations have been defined as multi-point correlations between a probe particle and a reference "set" of particles [78, 79, 224]. In simulations, the reference set of particles is normally realised through the freezing (pinning) of a suitable collection of particles, selected from an equilibrated configuration of the system [225]. The probability to find the probe particle at a certain relative position with respect to the set is then evaluated, and a corresponding static length $\xi_{\text {PTS }}$ can be defined [226].

Even if the method does not allow us to equilibrate the system more rapidly at lower temperatures, it has been shown that via the pinning of some sets of particles it is possible to easily access a critical-like regime at relatively high temperatures. In this regime, the degree of similarity between different configurations (as measured by the so-called overlap order parameter) fluctuates and a first-order, equilibrium phase transition between configurations with low and high overlap can be detected. Within the framework of Random First Order Transition theory (RFOT) [9], this transition can be seen as reminiscent of a low-temperature, inaccessible, thermodynamic transition from the supercooled-liquid to an "ideal" glass, with configurational entropy comparable to

that of a crystal, but devoid of long-range order. In this sense, pinning would allow us to access, at higher temperatures, a high-overlap phase which would be representative of the kind of amorphous phase associated with the "ideal" glass phase. It is implicit to observe that such a high overlap phase is also a phase with very low mobility.

In practice, two methods have been followed in order to measure the point-to-set correlation lengths $\xi_{\mathrm{PTS}}$ :

Cavity pinning. - In an equilibrated liquid configuration a cavity of a given radius $R$ can be identified, outside which all particles are pinned. The pinning of the particles on the edge of the cavity reduces the configurational entropy of those inside, biasing the system in favour of arrest. If the radius $R$ of the cavity is larger than $\xi_{\text {PTS }}$, the particles at the centre remain mobile, if the cavity is smaller than $\xi_{\mathrm{PTS}}$, the entire cavity is immobilised (Fig. 13 insets) [226, 91, 227].

Random pinning - Rather than pinning all particles outside a cavity, one can instead pin a certain proportion throughout the system [228, 229]. The concentration of 
a

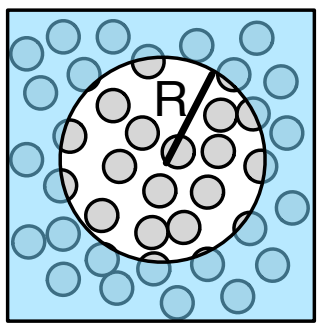

b

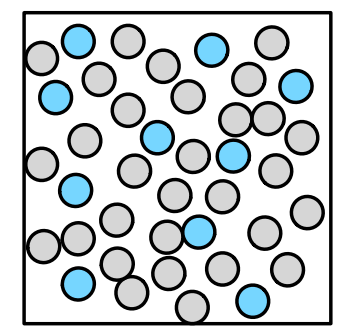

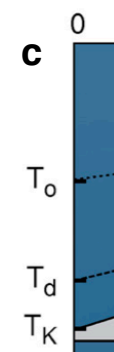

$T_{d}^{M F}$
$T_{h}$
$T_{K}$
0
0.05

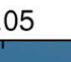

0.1

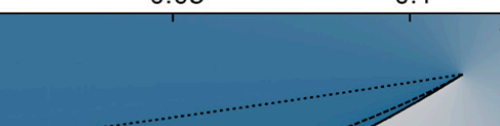

$T_{K}(c)$

$T_{0}(c)$

$-0.70$

0.65

0.60

Figure 14. Pinning techniques. (a) Particles outside a region of radius $R$ are frozen in cavity pinning. In random pinning (b) an arbitrary concentration $c$ of particles is pinned (light blue) from an equilibrium configuration. Reproduced with permission from J. Chem. Phys. 131, 194901 (2008). Copyright 2008 American Chemical Society. (c) Temperature vs pinning concentration $c$ phase diagram in the p-spin model from mean field (top) and renormalization group calculations (bottom): marked are the glassy dynamics onset temperature $T_{o}(c)$, the Mode-Coupling critical temperature $T_{d}(c)$, the ideal glass transition temperature $T_{K}(c)$. The phase diagram presents a coexistence line (continuous curve) distinguishing a high overlap form a low overlap region. The line starts at $T_{K}$ for $c=0$ and terminates at a critical point at $T_{h}$. Reproduced from [81.

this pinned population of particles $c$ leads to a mean separation $\ell_{c}=c^{-1 / d}$ (where $d$ is dimension). When $\ell_{c}<\xi_{\text {PTS }}$, it is argued that an ideal glass transition corresponding to that predicted by RFOT around $T_{K}$ is found [81, 82, 230]. It is worth noting that pinned configurations are (with sufficient sampling) formally structurally indistinguishable from their unpinned counterparts at the same temperature $\mathbb{\Psi}$. Nevertheless increasing the concentration of pinned particles means that the configurational entropy drops and strong many-body correlations are established [232]. Recently, experimental evidence has been found for dynamical arrest upon pinning using colloidal suspensions (see Section 3.5] 165, 161, 160].

\subsection{Simulated vapor deposition}

Via Molecular Dynamics and kinetic Monte-Carlo techniques it is possible to reproduce in silico the physical process of vapor deposition of simple model systems. These methods have originally been developed to study phenomena such as condensation

I Crudely speaking this can be seen from the fact that the pinned system interacts via the same Hamiltonian as does the unpinned system, and that pinned particles are chosen to be frozen in an equilibrium supercooled liquid [231]. 
on surfaces [233], epitaxial growth [234] or the properties of thin films such as diamond [235, 236] and graphene [237] from the material science point of view.

Recently, prompted by the achievements in experimentally deposited glasses (see section 3.3), these methods have been applied to produce ultrastable states for model glass formers. The simulation protocol is designed to reproduce the physics of experiments: a substrate is thermalised separately from vapor molecules that are gradually deposited at random locations and orientations over it; after energy minimization and a short equilibration run, the procedure is reiterated so that a thick film of deposited particles can be grown.

Glasses prepared through vapor deposition acquire a higher kinetic stability due to the higher density that they manage to achieve [238, 239, 240, 241]. In simulations of polymeric glasses, it has been shown [242] that while such increased stability is correlated with a high degree of anisotropy in the final structure of glasses formed by long polymeric chains (with evident layering in the direction orthogonal to the substrate), short polymer chains still access very low energy regions of the energy landscape and remain isotropic. Results, for small molecules, which relate the nature of the orientation to the substrate temperature, reproduce experimental results of anisotropic glasses in the form of laterally oriented molecules for very low temperatures and vertically oriented molecules for temperatures mildly below the glass transition where ultrastable glasses teaches their optimal fictive temperature [140].

For models with spherically symmetric interactions, vapor deposition is equally successful and generates isotropic glasses that are as low in energy as glasses obtained from cooling rates that are 2-3 orders of magnitude lower than those used in ordinary simulations of glasses [243]. While the structures that are formed in vapour-deposited systems are statistically identical to those observed in ordinary glasses compared at the same inherent state energy [244], compositional inhomogeneities can be measured at the interfaces of the films formed by vapor deposition [241, 244]. Recently, computer simulations of a polydisperse Lennard-Jones model [245] provided further evidence for the idea that the increased kinetic stability as a consequence of the enhanced surface diffusion of vapor deposited glasses, suggesting a possible explanation of the link between the experimentally observed surface mobility [63, 246] and the properties of these

ultrastable glasses.

\subsection{Large deviations of time-integrated observables}

As previously mentioned, very low temperature (and low free energy), supercooled liquids are characterised by strong dynamical heterogeneities [21]. In particular, dynamical arrest corresponds to the growth of large regions where the particle motion is suppressed and only rare thermally activated processes succeed in triggering some mobility.

It is possible to provide a quantitative spatio-temporal description of dynamical heterogeneities with the use of space and time integrated physical observables, defined 
over realisations or trajectories (also called histories) of a system. Within the framework of large deviation theory applied to non-equilibrium systems [247, 248, 249, 250], free energies for trajectory-dependent observables have been defined, which led to the determination of phase transitions in trajectory space for model systems of glassy dynamics [11, 168, 251, 252, 253, 254]. Such phase transitions depend on the choice of the time-extensive observable under consideration: oftentimes, it is the activity (i.e. the particle mobility) that it is considered [252]; for systems under external forces such as shear, the total particle current has been studied [255]; when structural information is at hand, it is also possible to compute time integrals of the concentration of welldefined structural motifs and arrangements [168]. In all cases, it appears that, for temperatures below the onset temperature, schematic and atomistic models of glassy dynamics present the signature of a dynamical phase coexistence between trajectories with high mobility/high currents/low concentration of structures and low mobility/low currents/high concentration of structure. While the former characterises high energy liquid states, the latter can be seen as representative of low temperature arrested states.

The approach in terms of large deviations of time-integrated observables is inherently dynamical. Connecting such a dynamical description to the thermodynamics of supercooled liquids is a challenging and ongoing research direction [256]. In the following we discuss in more detail two complementary approaches to explored dynamical phase transition in glassy systems: (a) the measurement of activity (i.e. time integrated mobility) and its fluctuations in the so-called s-ensemble; (b) the measurement of the time-integrated density of locally favoured structures and its fluctuations, the so-called $\mu$-ensemble.

The s-ensemble In the context of dynamical arrest, dynamical phase transitions have been first identified in simple models with trivial energetics but non-trivial dynamical rules that mimic steric hindrance and jamming, i.e. kinetically constrained models [257]. Chandler and Garrahan and coworkers [251, 253] tracked the activity per trajectory (total particle dispacements or spin flips) of particles (or spins) on one and two dimensional lattices and computed its probability distribution through the generation of many trajectories of the system [258, 259]. At low enough temperatures, the probability distribution shows non-gaussian tails, with slow, inactive trajectories being over-represented. Such "fat tails" in the distribution are akin to a thermodynamic transition in a small system (say the liquid-vapour transition) where the system samples configurations from both phases with some probability and where one controls temperature to approach phase transitions and critical points. Within the framework of large deviation theory one can show that the formal analog of the thermodynamic temperature is the conjugated field $s$. In general terms, it is possible to write that the partition function $Z_{C_{\tau}}(s)$ for trajectories of length $\tau$ characterised by the total activity $C_{\tau}$ can be obtained from the probability distribution as

$$
Z_{C_{\tau}}(s)=\sum_{\text {trajectories }} \operatorname{Prob}(\text { trajectory }) e^{-s C_{\tau} \text { (trajectory) }}
$$


where $s$ is a field conjugated to the activity $C_{\tau}$. This is reminiscent of the equilibrium partition function $Z=\sum_{\text {configurations }} \operatorname{Prob}$ (configuration) $e^{-E / T}$ where $T$ is the temperature measured in units of the Boltzmann constant. This construction allows us to build a canonical ensemble of trajectories, conventionally named s-ensemble. Fluctuations from the average activity correspond to particular values of the field $s$ :

$$
\left\langle C_{\tau}\right\rangle_{s}=\sum_{\text {trajectories }} \operatorname{Prob}(\text { trajectory }) e^{-s C_{\tau}} C_{\tau} / Z_{C_{\tau}}
$$

with the typical average value $\bar{C}_{\tau} \equiv \sum_{\text {trajectories }}$ Prob(trajectory) $C_{\tau} / Z_{C_{\tau}}=\left\langle C_{\tau}\right\rangle_{s=0}$ corresponding to $s=0$. We remark, however, that in the case of trajectory sampling the thermostatting temperature $T$ is not varied (it is typically chosen not too low in order to enhance sampling) because the focus is on fluctuations of time-integrated observables a-priori unrelated to the temperature.

This framework allowed Chandler and Garrahan and co-workers 252] to identify a phase transition at a coexistence value $s^{*} \neq 0$ between an active phase with high mobility and an inactive phase with subextensive mobility. They used small systems ( $N \sim 100$ particles) of the Kob-Andersen binary mixture to demonstrate a first-order dynamical phase transition in trajectory space. In this dynamical ensemble, rather than density (liquid or vapour), it is the fraction of suitably defined active (mobile) particles per trajectory $c=C_{\tau} / N$ of the trajectories which identifies the two "phases". The distribution in Fig. 15(a) shows that low-mobility trajectories are much more likely than expected: this is taken as evidence for an "inactive phase". The implication is that dynamical heterogeneity is a manifestation of these two phases, fluctuating in and out of existence like density fluctuations in liquid-vapour critical phenomena.

The $\mu$-ensemble A formally analogous construction can be achieved if, instead of the particle activity $A_{\tau}$ on trajectories of length $\tau$, one takes the time-integrated number of particles in locally favoured structures $\mathcal{N}_{\tau}=\sum_{t=0}^{\tau} N n_{\mathrm{LFS}}(t)$ where $n_{\mathrm{LFS}}(t)$ is the concentration of locally favoured structures (LFS) in a configuration at time $t$. Locally favoured structures are recurrent local motifs whose concentration appears to increase as a glass-former is cooled down and which have been identified with emergence of slow dynamics for some systems such as hard spheres and discs or Lennard-Jones binary mixtures and metallic glassformers [170]. The canonical ensemble of trajectories with average number of locally favoured structures per trajectory $\overline{\mathcal{N}}$ is associated to a conjugated field termed $\mu$. Again, $\mu$ is related to fluctuations from the typical value $\overline{\mathcal{N}}=\langle\mathcal{N}\rangle_{\mu=0}$ obtained at $\mu=0$, so that nonzero values of $\mu$ quantify how atypical it is to observe trajectories with time-integrated structural observable $\mathcal{N}(\mu)$.

Speck et al. demonstrated [168] that the transition in the $s$-ensemble (related to a purely dynamical observables such as the particle mobility) has its counterpart in the structural description realised in the $\mu$-ensemble: inactive trajectories correlate strongly with trajectories rich in locally favoured structures (see Fig. 15). Experimental evidence, using colloidal suspensions, has now been found for this dynamical phase transition (see Section 3.5 and Fig. 10] [?]. More recent studies [256] suggest that the inactive/locally 

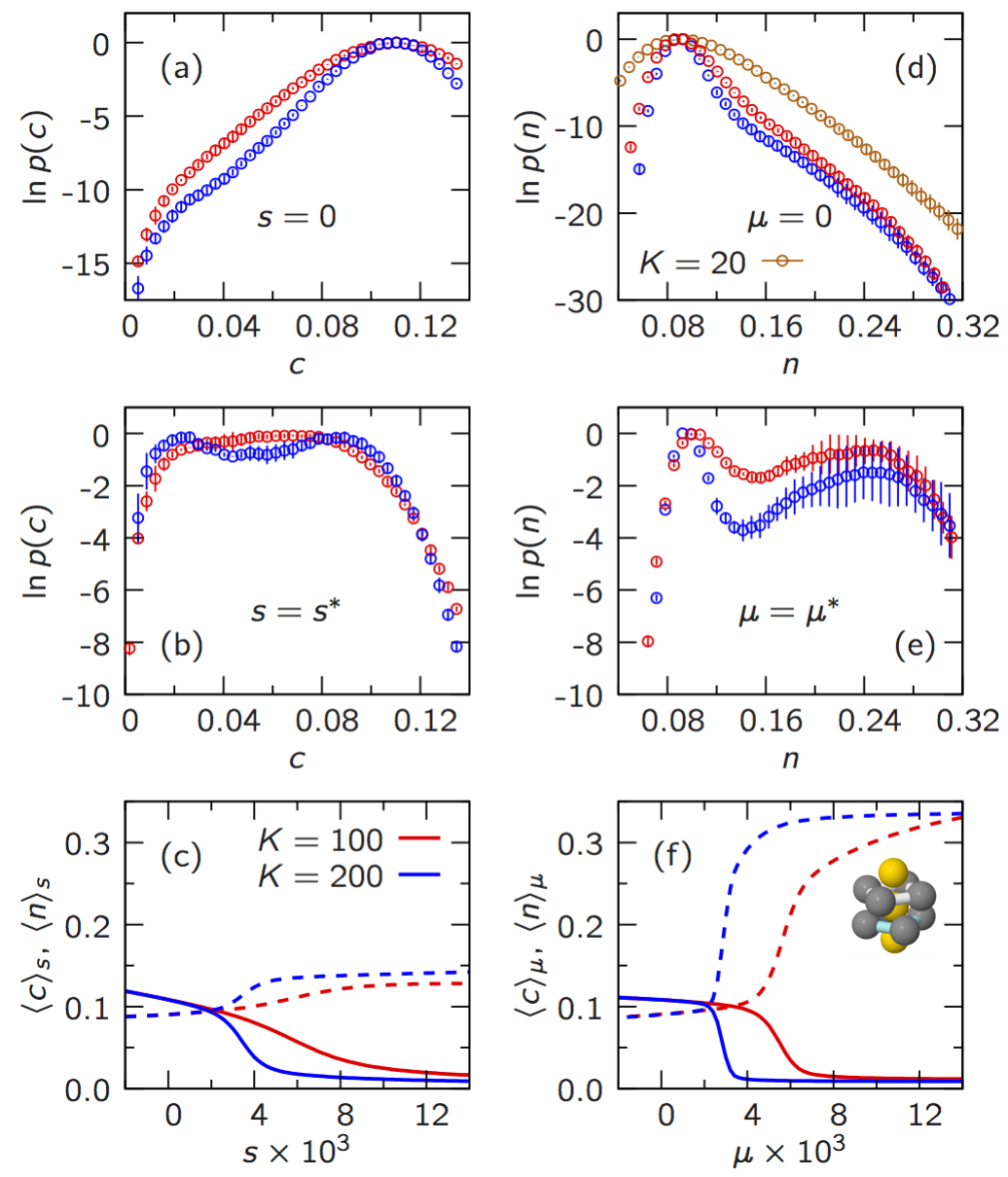

Figure 15. Phase transitions in trajectory space for the Kob-Andersen binary Lennard-Jones mixture (reproduced from [168]). Left column: $s$-ensemble (a) Probability distributions $p(c)$ for the density of mobile particles $c$ for two trajectory lengths. The non-concave shape indicates a phase transition in trajectory space as becomes obvious from the bimodal distribution (b) at the field $s^{*}$ that maximises the fluctuations $\left\langle c^{2}{ }_{s}\right\rangle-\left\langle c_{s}\right\rangle^{2}$. (c) Average fractions of mobile particles (solid lines) and bicapped square antiprism cluster population (dashed lines) vs. the biasing field $s$. Right column: (d-f) as left column but for the $\mu$-ensemble. Here the bicapped sqaure anitprism (depicted in (f)) is the locally favoured structure. Throughout, red and blue lines refer to $K=100$ and $K=200$, respectively. $K$ denotes the length of the trajectory. Here $K \approx 0.2 \tau_{\alpha}[168$.

favoured structure-rich phase obtained in the space of trajectories also correlates strongly with particularly low (potential) energy states, and that when decreasing the temperature, the inactive, LFS-rich phases tend to dominate the statistics, with the coexistence values of $s^{*}$ and $\mu^{*}$ approaching zero as the temperature is decreased. In this sense, guiding trajectory sampling with the usage of time-integrated observable can be an efficient way to identify low energy states, more present in the arrested glassy phases.

These ideas have been recently tested in the case of a canonical atomistic glass former (the Kob-Andersen binary mixture) [125], and it has been shown that other 
(a)

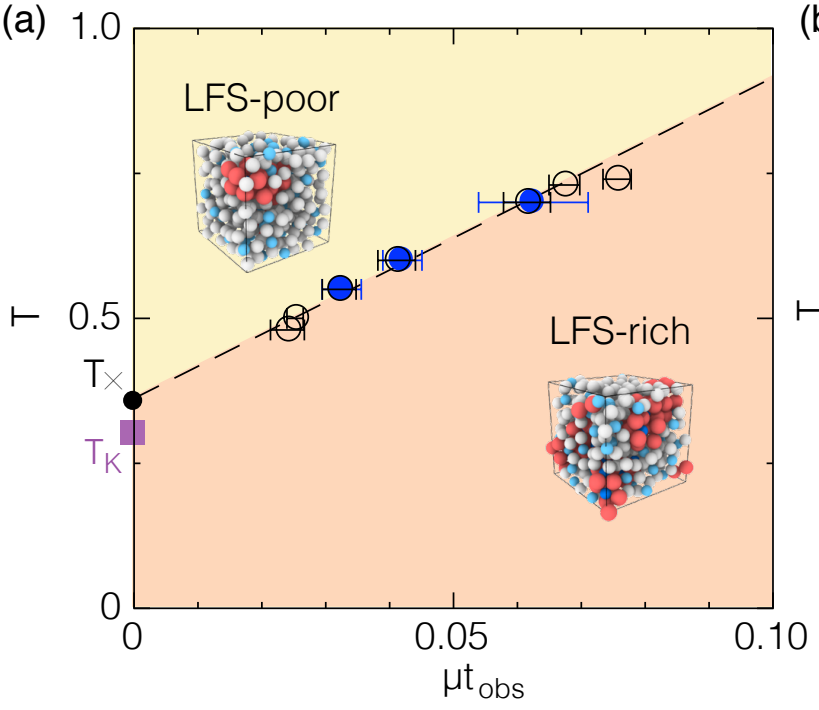

(b)

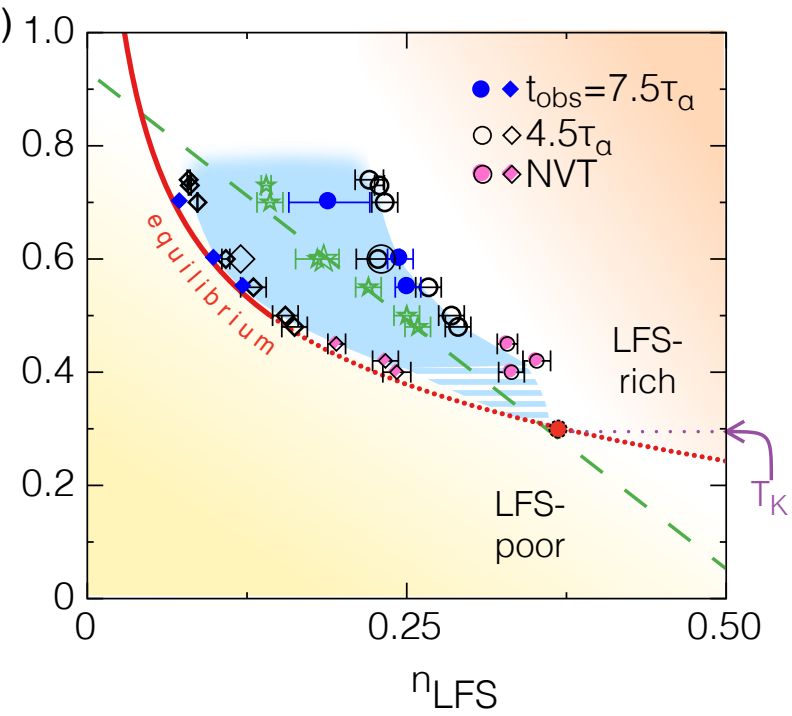

Figure 16. Results of trajectory sampling ( $\mu$-ensemble) computer simulations of the Kob-Andersen binary Lennard-Jones mixture. (a) Temperature versus $\mu$ phase diagram. Two distinct structural-dynamical phases are found at coexistence at a finite value $\mu^{*}$ of the field $\mu$ when sampling trajectories of different durations $t_{\text {obs }}$ (filled and empty circles): these are poor and rich in structure (LFS poor/rich), represented in the insets (with red and dark blue particles indicating the LFS regions). The scaled value $\mu^{*} t_{\mathrm{obs}}$, however, lies on a single curve. A linear extrapolation (dashed line) indicates that at a temperature $T_{\times} \approx T_{\mathrm{K}}$ one would observe the transition from one phase to the other directly in the thermal average of structural quantities $(\mu=0)$ without recurring to large deviations, under the form of intermittency. (b) Dynamical coexistence in the temperature versus concentration of LFS per trajectory plane. The coexistence region (determined by several numerical methods, in blue) has a non-trivial temperature dependence and narrows as the temperature is reduced. The equilibrium supercooled liquid approaches the coexistence region gradually and is always located close to the LFS-poor boundary. The extrapolation of the line of susceptibility maxima (green stars) and the equilibrium line meet at a temperature close to $T_{\mathrm{K}}$, suggesting a cross-over between the LFS-poor and the LFS-liquid. More information in [125].

systems exhibit similar behaviour, such as hard spheres [?] and the Wanhström binary Lennard-Jones model [260]. In particular, it has been shown that the large deviations of time-integrated structural observables give access to low energy configurations that sample the tails of the probability distribution of inherent state energies. Through reweighting, this sampling recovers the thermodynamical properties of the system (such as the configurational entropy) down to very low temperatures, without the need of sampling the dynamics at low temperatures directly.

Additionally, this approach shows that the dynamical phase transition between trajectories poor/rich in structure sampled in the trajectory ensemble corresponds to a transition between two distinct metastable amorphous states at high/low inherent state energies respectively: one corresponds to the supercooled liquid sampled in conventional dynamics; the other to a secondary amorphous state, with low energy, low configurational entropy, rich in structure and very slow dynamics, see Fig. 16. This 
second amorphous state is more metastable than the conventional supercooled liquid: however, the difference in stability (as measured by the value $\mu^{*}$ of the conjugated field $\mu$ at coexistence between the two phases) is a function of the temperature and decreases as the temperature is reduced. Extrapolations indicate that $\mu^{*}(T) \rightarrow 0$ at a finite temperature $T_{\times} \approx T_{\mathrm{K}}$.

This is suggestive, as it indicates the possibility of an alternative interpretation of the ideal glass transition temperature $T_{\mathrm{K}}$ : at such low temperatures the structure of the liquid would change (in a continuous, or weakly discontinuous fashion) from a liquid poor in local structure to a liquid rich in structure. Such liquid-to-liquid transition would be an alternative solution of Kauzmann's original paradox, as the structure-rich liquid (whose configurational entropy is weakly dependent on the temperature, according to the simulations) would behave as a strong liquid with divergence of the relaxation times only at temperature $T=0$. We should note that these conclusions are somewhat speculative. The work suggests that the dynamical phase transition has a lower critical point close to the Kauzmann temperature. But proximity alone doesn't necessarily mean the two are connected. We discuss other scenarios for the fate of the supercooled liquid in the next section.

\section{What does it all mean?}

Finally we consider what we have learnt. We began this article by noting that dynamical arrest is a major challenge and there is no firmly agreed paradigm for the phenomenon. The issue lies in understanding the behaviour of glassforming systems in the supercooling regime between the molecular glass transition $T_{g}$ and the Kauzmann temperature $T_{K}$ ( or $T_{0}$ ). One might imagine that the kind of studies outlined here, namely attempts to equilibrate glassformers in this very deep range of supercooling might shed light on the nature of the glass transition. The first observation we might make is that it appears possible to supercool a system through $T_{g}$. In terms of experiments on molecules, which have the potential to reach states very low in the energy landscape, there is evidence that the configurational entropy continues to drop. This is - or should be - found in the work on ultrastable glasses.

In the case of numerical work, pinning (section 4.3), particle swaps (section 4.2) and dynamical phase transitions (section 4.5) have certainly found considerable evidence in support of a drop in configurational entropy [261. This would tend to support thermodynamic theories such as Adam-Gibbs/Random First Order Transition theory. Other examples include the large deviations approach of Turci et al. [125] which indicates a lower critical point of the dynamical transition related to dynamic facilitation theory around the Kauzmann temperature. The coming together of the equilibrium liquid and low-entropy non-equilibrium phase related to the dynamical transition suggests a possible means of unification of the large deviations approach

with thermodynamic theories of Adam-Gibbs/Random First Order Transition theory [7, 9] and perhaps even geometric frustration with a transition to a local structure-rich 


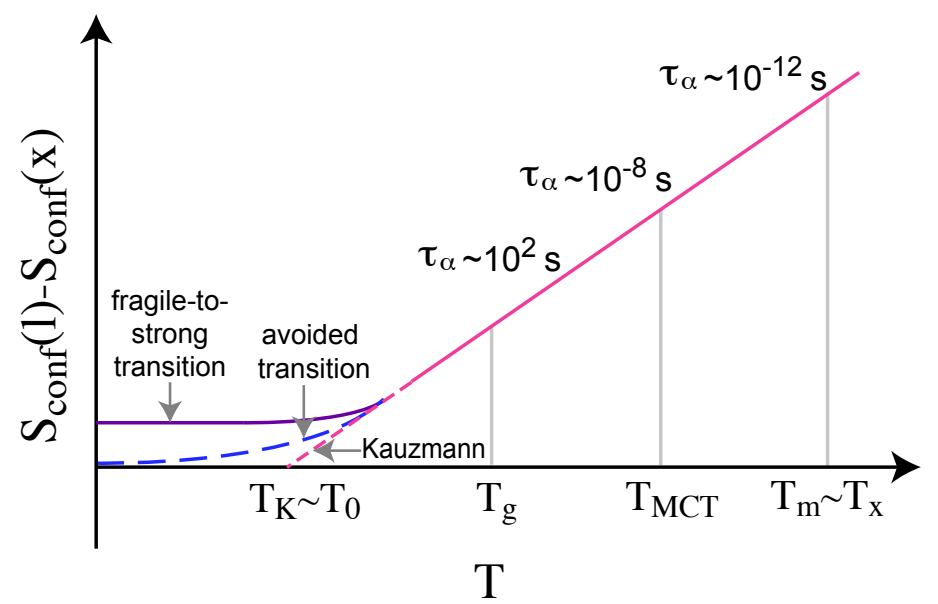

Figure 17. The decrease of the liquid configurational entropy with respect to that of the crystal. Here $S_{\text {conf }}(l)$ and $S_{\text {conf }}(r)$ are the liquid and crystal configurational entropies respectively. Three scenarios are depicted. The short dashed line is the original Kauzmann extrapolation [34, the long dashed line pertains to the suggestion by Stillinger et al. that any ideal glass would have "defects" and thus the transition would be smoothed or avoided [36] and the solid line is a possible fragile-to-strong transition.

state albeit dynamical, rather than the avoided more conventional transition [125].

What can we deduce about the nature - or existence - or otherwise of the ideal glass, or Kauzmann transition of the Adam-Gibbs/RFOT approach? While of course this remains speculative, it is clear that, should such a transition exist, the Kauzmann point has been approached much closer than before. However, there is one piece of information lacking from much of the work which has been carried out - and for good reason: the dynamics of such deeply supercooled materials are incredibly hard to measure. The result of not knowing the relaxation time often means we must extrapolate from higher temperature, assuming a constant fragility, with little or no justification. This means that dynamical data obtained indirectly are important. Notably two means to extract dynamical information in this regime we have mentioned above both indicate an Arrhenius behaviour in the regime of interest, $T<T_{g}$ sections 3.2 [119] and 3.3 [147].

Now such fragile-to-strong transitions have been noted before [262, 263, 170], and may be related to the system reaching the bottom of its energy landscape yet somehow stopping short of the "ideal" glass, so that the Kauzmann transition is not reached 262. In this picture the configurational entropy would then be essentially constant with respect to temperature, i.e. the structure of the system is independent of temperature. This would suggest an extensive configurational entropy for all non-zero temperatures, as indicated in Fig. 17.

Such arguments relate to work by Stillinger et al. [36], which are indicated in Fig. 17. Stillinger et al. noted that that thermal motion would lead to a finite population of "defects" in the otherwise "ideal" glass, thus making the transition to any ideal 
glass into a crossover at best. This would lead to a finite relaxation time. Indeed, if this "defect population" were somehow constant over some temperature range, even Arrhenius behaviour might be found.

Perspectives. - After the above arguments, it may seem that we are little closer to resolving the origin of the glass transition. However, while the origin of the slow dynamics remains out of reach for now, it feels tantalisingly close. We close with the following observations.

- Adam-Gibbs/RFOT-like behaviour is found until very close to the point at which the Kauzmann transition is predicted.

- It seems possible to unify the seemingly different approaches of dynamic facilitation and Adam-Giibs/RFOT via the discovery of a possible lower critical point to the dynamical phase transition close to or coincident with the Kauzmann temperature.

- If any insight can be gained as to the dynamics in the new, deeply supercooled simulation data, it would be most useful. The methods of Pogna et al. [147] might be taken as a starting point, though the challenges of obtaining suitable data are hard to overstate.

- Some means to identify "defects" such as those postulated by Stillinger et al. 36] might provide another route to deducing which scenario in Fig. 17 holds. Some attempt to identify defects in the form of "locally unfavoured structures" has been made [51, 90], but much remains to be done. Order agnostic approaches such as overlap may provide crucial insight here [230].

- If we can somehow deduce the dynamics, then the more ambitious task of identifying dynamic lengthscales beyond $T_{g}$ may well provide key evidence to discriminate between competing theoretical approaches.

Thus we argue that the remarkable progress made in the last few years towards the bottom of the energy landscape sets us up to refine the approach we might take towards resolving the existence - or otherwise - of the ideal glass. In particular we believe that the key lies in probing dynamics, and if possible, dynamic lengthscales, and "defects".

\section{Acknowledgments}

In connection with the preparation of this article, the authors would like to thank Chistiane Alba-Simionesco, Austen Angell, Ludovic Berthier, Giulio Biroli, Chiara Cammarota, David Chandler, Patrick Charbonneau, Eric Corwin, Daniele Coslovich, Olivier Dauchot, Jeppe Dyre, Mark Ediger, Juan P. Garrahan, Peter Harrowell, Trond Ingebrigsten, Rob P. Jack, Walter Kob, Andrea Liu, Tannie Liverpool, Kuni Miyazaki, Mike Moore, Sid Nagel, Ross Poldark, Itamar Procaccia, Tulio Sciopignio, 
Francesco Sciortino, Thomas Speck, Grzegorz Szamel, Hajime Tanaka, Gilles Tarjus, David Wales, Eric Weeks, Stephen Williams, Mathieu Wyart, Hai-Bin Yu, Emanuela Zaccarelli, and Francesco Zamponi for helpful discussions. We thank Ludovic Berthier, Daniele Coslovich, Patrick Charbonneau and Mark Ediger for helpful comments on the manuscript. Rattachai Pinchaipat and Sho Yaida are gratefully acknowledged for providing data. Chiharu Nakamura is thanked for the bally-ball stuff. CPR gratefully acknowledges the Royal Society, European Research Council (ERC Consolidator Grant NANOPRS, project number 617266) and Kyoto University SPIRITS fund for financial support.

[1] J C Mauro and M M Smedskjaer. Statistical mechanics of glass. J. Non-Cryst. Sol., 396-397:41$53,2014$.

[2] M. D. Ediger. Perspective on high stability vapor-deposited glasses. submitted to J. Chem. Phys., 2017.

[3] D Lencer, M Salinga, B J Grabowski, T Hickel, J Neugebauer, and M Wuttig. A map for phase-change materials. Nature. Mater., 7:972-977, 2008.

[4] M Wuttig and N Yamada. Phase-change materials for rewriteable data storage. Nat. Mater., 6:824-832, 2007.

[5] L Berthier and G Biroli. Theoretical perspective on the glass transition and amorphous materials. Rev. Mod. Phys., 83(2):587-645, 2011.

[6] A Cavagna. Supercooled liquids for pedestrians. Phys. Rep., 476:51-124, 2009.

[7] G Adam and J H Gibbs. On the temperature dependence of relaxation phenomena in glassforming liquids. J. Chem. Phys., 43:139-146, 1965.

[8] M Goldstein. Viscous liquids and the glass transition : a potential energy landscape picture. $J$. Chem. Phys., 51:3728-3739, 1969.

[9] V Lubchenko and P Wolynes. Theory of Structural Glasses and Supercooled Liquids. Ann. Rev. Phys. Chem., 58:235-266, 2007.

[10] G Tarjus, S a. Kivelson, Z Nussinov, and P Viot. The frustration-based approach of supercooled liquids and the glass transition: a review and critical assessment. J. Phys. Condens. Matter, 17(50):R1143-R1182, 2005.

[11] D Chandler and J P Garrahan. Dynamics on the way to forming glass: bubbles in space-time. Ann. Rev. Phys. Chem., 61:191-217, jan 2010.

[12] P G Debenedetti. Metastable liquids. Princeton University Press, Princeton, NJ, 1996.

[13] Jeppe C Dyre. Colloquium: The glass transition and elastic models of glass-forming liquids. Rev. Mod. Phys., 78(3):953-972, sep 2006.

[14] F H Stillinger and P G Debenedetti. Glass Transition Thermodynamics and Kinetics. Annu. Rev. Condens. Matter Phys., 4:263-285, 2013.

[15] L Berthier and M D Ediger. Facets of glass physics. Phys. Today, 69:40, 2016.

[16] G Biroli and J P Garrahan. Perspective: The Glass Transition. J. Chem. Phys., 138:12A301, mar 2013.

[17] P G Debenedetti and F H Stillinger. Supercooled liquids and the glass transition. Nature, 410(6825):259-267, mar 2001.

[18] M D Ediger and P Harrowell. Perspective: Supercooled liquids and glasses. J. Chem. Phys., 137:80901, 2012.

[19] F Sciortino. Potential energy landscape description of supercooled liquids and glasses. J. Stat. Mech Theory Exp., page P05015, 2005.

[20] A Heuer. Exploring the potential energy landscape of glass-forming systems: from inherent structures via metabasins to macroscopic transport. J. Phys Condens. Matter, 20:373101, 2008.

[21] L Berthier, G Biroli, J P Bouchaud, L Cipelletti, and W Van Saarloos. Dynamical heterogeneities 
in glasses, colloids, and granular media, volume 150. Oxford Univ. Press, 2011.

[22] M D Ediger. Spatially heterogeneous dynamics in supercooled liquids. Annu. Rev. Phys. Chem., 51:99-128, 2000.

[23] P Charbonneau and D Reichman. Mode-coupling theory. J. Stat. Mech Theory Exp., pages $1-22,2005$.

[24] W Goetze. Complex Dynamics of Glass-Forming Liquids: A Mode-Coupling Theory. International Series of Monographs on Physics. Oxford Univ. Press, 2009.

[25] P. Charbonneau, J. Kurchan, G. Parisi, P. Urbani, and F. Zamponi. Glass and jamming transitions: From exact results to finite-dimensional descriptions. Ann. Rev. Condens, Matt. Phys., 8:265-288, 2017.

[26] G Parisi and F Zamponi. Mean-field theory of hard sphere glasses and jamming. Rev. Mod. Phys., 82(1):789, 2010.

[27] P. K. Gupta and J. C. Mauro. Composition dependence of glass transition temperature and fragility. i. a topological model incorporating temperature-dependent constraints. $J$ Chem. Phys., 130:094503, 2009.

[28] P Sollich. Molecular Gels: Materials with Self-Assembled Fibrillar Networks, chapter 5, Soft glassy rheology, pages 161-192. Springer, 2006.

[29] G. B. Mckenna and S. L. Simon. 50th anniversary perspective: Challenges in the dynamics and kinetics of glass-forming polymers. Macromolecules, 50:6333-6361, 2017.

[30] Y Q. Cheng and E Ma. Atomic-level structure and structure-property relationship in metallic glasses. Prog. Mater. Sci., 56(4):379-473, 2011.

[31] K L Kearns, S F Swallen, M D Ediger, T Wu, Y Sun, and L Yu. Hiking down the Energy Landscape: Progress Toward the Kauzmann Temperature via Vapor Deposition. J. Phys. Chem. B, 112(16):4934-4942, apr 2008.

[32] S L L M Ramos, M Oguni, K Ishii, and H Nakayama. Character of Devitrification, Viewed from Enthalpic Paths, of the Vapor-Deposited Ethylbenzene Glasses. J. Phys. Chem. B, 115(49):14327-14332, 2011.

[33] S Tatsumi, S Aso, and O Yamamuro. Thermodynamic study of simple molecular glasses: Universal features in their heat capacity and the size of the cooperatively rearranging regions. Phys. Rev. Lett., 109(4):045701, jul 2012.

[34] W Kauzmann. The Nature of the Glassy State and the Behavior of Liquids at Low Temperatures. Chem. Rev., 43(2):219-256, oct 1948.

[35] H. Tanaka. Possible resolution of the kauzmann paradox in supercooled liquids. Phys. Rev. E., 68:011505, 2003.

[36] F H. Stillinger, P G. Debenedetti, and T M. Truskett. The kauzmann paradox revisited. J. Phys. Chem, B, 105(47):11809-11816, 2001.

[37] H. J. Hoffmann. Energy and entropy of crystals, melts and glasses or what is wrong in kauzmann paradox? Mat.-wiss. u.Werkstofftech, 43:528-533, 2012.

[38] F. Aliotta, R. C. Ponterio, F. Saija, and P. V. Giaquinta. Kovacs effect and the relation between glasses and supercooled liquids. ArXiV, page 1711.05489, 2017.

[39] F Sciortino, W Kob, and P Tartaglia. Inherent Structure Entropy of Supercooled Liquids. Phys. Rev. Lett., 83:3214-3217, 1999.

[40] C. A. Angell and S. Borick. Specific heats $c_{p}, c_{v}, c_{\text {conf }}$ and energy landscapes of glassforming liquids. J. Non-Cryst. Sol., 307-310:393-406, 2002.

[41] L. Berthier, P. Charbonneau, D. Coslovich, A. Ninarello, M. Ozawa, and S. Yaida. Configurational entropy measurements in extremely supercooled liquids that break the glass ceiling. Proc. Nat. Acad. Sci., 114:11356-11361, 2017.

[42] G Biroli, J P Bouchaud, K Miyazaki, and D R Reichman. Inhomogenous Mode-Coupling Theory and Growing Dynamic Length in Supercooled Liquids. Phys. Rev. Lett., 97:195701, 2006.

[43] L M. C. Janssen and D Reichman. Microscopic dynamics of supercooled liquids from first principles. Phys. Rev. Lett., 115:205701, 2015. 
[44] S. M. Bhattacharyya, B. Bagchi, and P. G. Wolynes. Bridging the gap between the mode coupling and the random first order transition theories of structural relaxation in liquids. Phys. Rev. E., 72:031509, 2005.

[45] S. M. Bhattacharyya, B. Bagchi, and P. Wolynes. Facilitation, complexity growth, mode coupling, and activated dynamics in supercooled liquids. Proc. Nat. Acad. Sci., 105:16077-16082, 2008.

[46] P Charbonneau, J Kurchan, G Parisi, P Urbani, and F Zamponi. Fractal free energy landscapes in structural glasses. Nat. Comm., 5:3725, 2014.

[47] CA. A Angell. Structural instability and relaxation in liquid and glassy phases near the fragile liquid limit. J. Non. Cryst. Solids, 102(1-3):205-221, jun 1988.

[48] C A Angell. Formation of glasses from liquids and biopolymers. Science (80-. )., 267:1924-1935, 1995.

[49] L Berthier and T A Witten. Glass transition of dense fluids of hard and compressible spheres. Phys. Rev. E, 80:21502, 2009.

[50] L Berthier and G Tarjus. Nonperturbative effect of attractive forces in viscous liquids. Phys. Rev. Lett., 103:170601, 2009.

[51] C P. Royall and W Kob. Locally favoured structures and dynamic length scales in a simple glass-former. J. Stat. Mech.: Theory and Experiment, page 024001, 2017.

[52] J Robinson, F Turci, and C P. Royall. Josh and his amazing metallic glasses. in preparation, 2017.

[53] H-B. Yu, Yuansu Luo, and K Samwer. Ultrastable Metallic Glass. Adv. Mater., 25(41):59045908, nov 2013.

[54] C P. Royall, A Malins, A J. Dunleavy, and R Pinney. Strong geometric frustration in model glassformers. J. Non-Cryst. Solids, 407:34-43, 2015.

[55] L-M. Martinez and C A Angell. A thermodynamic connection to the fragility of glass-forming liquids. Nature, 410:663-667, 2001.

[56] H. Tanaka. Relation between thermodynamics and kinetics of glass-forming liquids. Phys. Rev. Lett., 90:055701, 2003.

[57] D. Cangialosi. Relationship between dynamics and thermodynamics in glass-forming polymers. EuroPhys. Lett., 70:614-620, 2005.

[58] T Hecksler, Albena I. Nielsen, N Boye Olsen, Jeppe C. Dyre, Tina Hecksher, Albena I. Nielsen, Niels Boye Olsen, and Jeppe C. Dyre. Little evidence for dynamic divergences in ultraviscous molecular liquids. Natue Phys., 4(9):737-741, sep 2008.

[59] J C Mauro, Y Yue, A J Ellison, P K Gupta, and D C Allan. Viscosity of glass-forming liquids. Proc. Nat. Acad. Sci., 106:19780, 2009.

[60] Y S Elmatad, D Chandler, and J P Garrahan. Corresponding States of Structural Glass Formers. J. Phys. Chem. B, 113:5563-5567, 2009.

[61] S. Mirigian and K. S. Schweizer. Unified theory of activated relaxation in liquids over 14 decades in time. J. Phys. Chem. Lett., 4:3648-3653, 2013.

[62] R. Pinney, T. B. Liverpool, and Royall C. P. Recasting a model atomistic glassformer as a system of icosahedra. J. Chem. Phys., 143:244507, 2015.

[63] S F Swallen, K L Kearns, M K Mapes, Y S Kim, R J McMahon, M D Ediger, T Wu, L Yu, and S Satija. Organic Glasses with Exceptional Thermodynamic and Kinetic Stability. Science (80-. )., 315(5810):353-356, 2007.

[64] D Perera and P Harrowell. Consequences of kinetic inhomogeneities in glasses. Phys. Rev. E, 54:1652-1662, 1996.

[65] W Kob, C Donati, S J Plimpton, P H Poole, and S C Glotzer. Dynamical heterogeneities in a supercooled Lennard-Jones liquid. Phys. Rev. Lett., 79:2827-2830, 1997.

[66] N Lačević, F W. Starr, T B. Schrøder, and S C. Glotzer. Spatially heterogeneous dynamics investigated via a time-dependent four-point density correlation function. J. Chem. Phys., 119(14):7372-7387, oct 2003.

[67] A Malins, J Eggers, C P Royall, S R Williams, and H Tanaka. Identification of long-lived clusters 
and their link to slow dynamics in a model glass former. J. Chem. Phys., 138:12A535, 2013.

[68] S Whitelam, L Berthier, and J-P. Garrahan. Dynamic criticality in glass-forming liquids. Phys. Rev. Lett., 92:185705, 2004.

[69] H Tanaka, T Kawasaki, H Shintani, and K Watanabe. Critical-like behaviour of glass-forming liquids. Nat. Mater., 9:324-331, 2010.

[70] E Flenner and G Szamel. Dynamic heterogeneities above and below the mode-coupling temperature: Evidence of a dynamic crossover. J. Chem. Phys., 138:12A523, 2013.

[71] E Donth. The size of cooperatively rearranging regions at the glass transition. J. Non. Cryst. Solids, 53(3):325-330, dec 1982.

[72] M T Cicerone and M D Ediger. Relaxation of spatially heterogeneous dynamic domains in supercooled orthoterphenyl. J. Chem. Phys., 103:5684-5892, 1995.

[73] L Berthier, G Biroli, J-P J.-P. Bouchaud, L Cipelletti, D El Masri, D L'Hôte, F Ladieu, M Pierno, D El Masri, D L'H^ ote, F Ladieu, and M Pierno. Direct experimental evidence of a growing length scale accompanying the glass transition. Science, 310(5755):1797-1800, 2005.

[74] U Tracht, M Wilhelm, A Heuer, H Feng, K Schmidt-Rohr, and H W Spiess. Length Scale of Dynamic Heterogeneities at the Glass Transition Determined by Multidimensional Nuclear Magnetic Resonance. Phys. Rev. Lett., 81(2727-2730), 1998.

[75] S Ashtekar, D Nguyen, K Zhao, J Lyding, W H Wang, and M Gruebele. An obligatory glass surface. J. Chem. Phys., 137:141102, 2012.

[76] C Dalle-Ferrier, C Thibierge, C Alba-Simionesco, L Berthier, G Biroli, J-P. Bouchaud, F Ladieu, D L'Hôte, and G Tarjus. Spatial correlations in the dynamics of glassforming liquids: Experimental determination of their temperature dependence. Phys. Rev. E, 76:41510, 2007.

[77] O Yamamuro, I Tsukushi, A Lindqvist, S Takahara, M Ishikawa, and T Matsuo. Calorimetric Study of Glassy and Liquid Toluene and Ethylbenzene: Thermodynamic Approach to Spatial Heterogeneity in Glass-Forming Molecular Liquids. J. Phys. Chem. B, 102(9):1605-1609, 1998.

[78] J P Bouchaud and G Biroli. On the Adam-Gibbs-Kirkpatrick-Thirumalai-Wolynes scenario for the viscosity increase in glasses. J. Chem. Phys., 121(15):7347, 2004.

[79] A Montanari and G Semerjian. Rigorous Inequalities between Length and Time Scales in Glassy Systems. J. Stat. Phys., 125:23-54, 2006.

[80] C Cammarota and G Biroli. Patch-repetition correlation length in glassy systems. Eur. Lett., 98:36005, 2012.

[81] C Cammarota and G Biroli. Ideal glass transitions by random pinning. Proc. Natl. Acad. Sci., 109(23):8850-8855, jun 2012.

[82] C. Cammarota and G. Biroli. Random pinning glass transition: Hallmarks, mean-field theory and renormalization group analysis. J. Chem. Phys., 138:12A547, 2013.

[83] G M Hocky, T E Markland, and D R Reichman. Growing point-to-set length scale correlates with growing relaxation times in model supercooled liquids. Phys. Rev. Lett., 108(22):225506, jun 2012.

[84] A J Dunleavy, K Wiesner, and C P Royall. Using mutual information to measure order in model glass-formers. Phys. Rev. E, 86:41505, 2012.

[85] A Malins, J Eggers, H Tanaka, and C P Royall. Lifetimes and lengthscales of Structural Motifs in a Model Glassformer. Faraday Discuss., 167:405-423, 2013.

[86] B Charbonneau, P Charbonneau, and G Tarjus. Geometrical Frustration and Static Correlations in a Simple Glass Former. Phy. Rev. Lett., 108:35701, 2012.

[87] P Charbonneau, B Charbonneau, G Tarjus, B Charbonneau, and G Tarjus. Geometrical frustration and static correlations in hard-sphere glass formers. J. Chem. Phys., 138(12):42305, 2013.

[88] P. Charbonneau and G. Tarjus. Decorrelation of the static and dynamic length scales in hardsphere glass formers. Phys. Rev. E, 87:042305, 2013.

[89] G M. Hocky, D Coslovich, A Ikeda, and D R. Reichman. Correlation of Local Order with Particle Mobility in Supercooled Liquids is Highly System Dependent. Phys. Rev. Lett., 113(15):157801, 
The race to the bottom: approaching the ideal glass?

2014.

[90] A J Dunleavy, K Wiesner, R Yamamoto, and C P Royall. Mutual information reveals multiple structural relaxation mechanisms in a model glassformer. Nat. Commun., 6:6089, 2015.

[91] P Charbonneau, E Dyer, J Lee, and S Yaida. Linking dynamical heterogeneity to static amorphous order. J. Stat. Mech.: Theory and Experiment, page 074004, 2016.

[92] K Kawasaki and H Tanaka. Structural origin of dynamic heterogeneity in three-dimensional colloidal glass formers and its link to crystal nucleation. J. Phys. Condens. Matter, 22:232102, 2010.

[93] M Leocmach and H Tanaka. Roles of icosahedral and crystal-like order in the hard spheres glass transition. Nat. Comm., 3:974, 2012.

[94] M Leocmach, J Russo, and Tanaka H. Importance of many-body correlations in glass transition: An example from polydispere hard spheres. J. Chem. Phys., 138:12A515, 2013.

[95] M Mosayebi, E Del Gado, P Ilg, and H C Öttinger. Probing a critical length scale at the glass transition. Phys. Rev. Lett., 104:205704, 2010.

[96] S Karmakar, C Dasgupta, and S Sastry. Growing Length Scales and Their Relation to Timescales in Glass-Forming Liquids. Annu. Rev. Cond. Matt. Phys., 5:255-284, 2014.

[97] P Harrowell. Dynamical heterogeneities in glasses, colloids, and granular media, chapter The length. Oxford Univ. Press, 2011.

[98] A Q Tool. Relation Between Inelastic Deformability and Thermal Expansion of Glass in Its Annealing Range. J. Am. Ceram. Soc., 29(9):240-253, sep 1946.

[99] O S Narayanaswarmy. A Model of Structural Relaxation in Glass. J. Am. Ceram. Soc., 54(10):491-498, 1971.

[100] C T. Moynihan, P B. Macedo, C J. Montrose, C J. Montrose, P K. Gupta, M A. DeBolt, J F. Dill, B E. Dom, P W. Drake, A J. Easteal, P B. Elterman, R P. Moeller, H Sasabe, and J A. Wilder. Structural Relaxation in Vitreous Materials. Ann. N. Y. Acad. Sci., 279(1 The Glass Tra):15-35, oct 1976 .

[101] Z Chen and R Richert. Dynamics of glass-forming liquids. XV. Dynamical features of molecular liquids that form ultra-stable glasses by vapor deposition. J. Chem. Phys., 135(12):124515, 2011.

[102] Y Z. Chua, M Ahrenberg, M Tylinski, M D. Ediger, and C Schick. How much time is needed to form a kinetically stable glass? AC calorimetric study of vapor-deposited glasses of ethylcyclohexane. J. Chem. Phys., 142(5), 2015.

[103] A Mandanici, W Huang, M Cutroni, and R Richert. Dynamics of glass-forming liquids. XII. Dielectric study of primary and secondary relaxations in ethylcyclohexane. J. Chem. Phys., 128(12), 2008.

[104] Sergio Luis L. M. Ramos, Atsuko K. Chigira, and Masaharu Oguni. Devitrification Properties of Vapor-Deposited Ethylcyclohexane Glasses and Interpretation of the Molecular Mechanism for Formation of Vapor-Deposited Glasses. J. Phys. Chem. B, 119(10):4076-4083, mar 2015.

[105] A Mandanici, W Huang, M Cutroni, and R Richert. On the features of the dielectric response of supercooled ethylcyclohexane. Philos. Mag., 88(33-35):3961-3971, 2008.

[106] J H Magill. Physical Properties of Aromatic Hydrocarbons. III. A Test of the Adam?Gibbs Relaxation Model for Glass Formers Based on the Heat-Capacity Data of 1,3,5-tri- $\alpha$ Naphthylbenzene. J. Chem. Phys., 47(8):2802, 1967.

[107] R Richert, K Duvvuri, and L T Duong. Dynamics of glass-forming liquids. VII. Dielectric relaxation of supercooled tris-naphthylbenzene, squalane, and decahydroisoquinoline. J. Chem. Phys., 118(4):1828-1836, 2003.

[108] A Dois, G Hinze, B Schiener, J Hemberger, and R Bhmer. Dielectric relaxation in the fragile viscous liquid state of toluene. J. Chem. Phys., 107(6):1740, 1997.

[109] A Sepúlveda, E Leon-Gutierrez, M Gonzalez-Silveira, C Rodríguez-Tinoco, M T. ClavagueraMora, and J Rodríguez-Viejo. Accelerated Aging in Ultrathin Films of a Molecular Glass Former. Phys. Rev. Lett., 107(2):025901, jul 2011. 
[110] S L Shamblin, X Tang, L Chang, B C Hancock, and M J Pikal. Characterization of the time scales of molecular motion in pharmaceutically important glasses. J. Phys. Chem. B, 103(20):41134121, 1999.

[111] Z Wojnarowska, K Adrjanowicz, P Wlodarczyk, E Kaminska, K Kaminski, K Grzybowska, R Wrzalik, M Paluch, and K L Ngai. Broadband dielectric relaxation study at ambient and elevated pressure of molecular dynamics of pharmaceutical: indomethacin. J. Phys. Chem. B, 113(37):12536-12545, 2009.

[112] Y Z. Chua, M Tylinski, S Tatsumi, M D. Ediger, and C Schick. Glass transition and stable glass formation of tetrachloromethane. J. Chem. Phys., 144(24), 2016.

[113] J Robinson. numerical calculation.

[114] L J Fetters, D J Lohse, R H Colby, and J E Mark. Physical Properties of Polymers Handbook. Springer New York, New York, NY, 2007.

[115] Y P Koh and S L Simon. Enthalpy Recovery of Polystyrene: Does a Long-Term Aging Plateau Exist? Macromolecules, 46(14):5815-5821, 2013.

[116] D Cangialosi, M Wübbenhorst, H Schut, A Van Veen, and S J. Picken. Dynamics of polycarbonate far below the glass transition temperature: A positron annihilation lifetime study. Phys. Rev. B - Condens. Matter Mater. Phys., 69(13):1-9, 2004.

[117] V M. Boucher, D Cangialosi, A Alegría, and J Colmenero. Enthalpy recovery of glassy polymers: Dramatic deviations from the extrapolated liquidlike behavior. Macromolecules, 44(20):83338342, 2011.

[118] J Zhao. Dynamics Below the Glass Transiiton Temperature and Viscoelastic and Calorimetric Investigation of Different Fossil Resins. PhD thesis, 2014.

[119] J Zhao, S L Simon, and G B McKenna. Using 20-million-year-old amber to test the superArrhenius behaviour of glass-forming systems. Nat. Commun., 4:1783, apr 2013.

[120] O Yamamuro and S Tatsumi. Thermodynamic Study on Simple Molecular Glasses. Nihon Reoroji Gakkaishi, 40(3):137-142, 2012.

[121] R Richert and C a Angell. Dynamics of glass-forming liquids. V. On the link between molecular dynamics and configurational entropy. J. Chem. Phys., 108(21):9016-9026, jun 1998.

[122] R Bohmer, K L Ngai, C A Angell, and D J Plazek. Nonexponential relaxations in strong and fragile glass formers. J. Chem. Phys., 99(5):4201, 1993.

[123] L Berthier, D Coslovich, A Ninarello, and M Ozawa. Equilibrium sampling of hard spheres up to the jamming density and beyond. Phys. Rev. Lett., 116(23):238002, 2016.

[124] J Taffs, S R Williams, H Tanaka, and C P Royall. Structure and kinetics in the freezing of nearly hard spheres. Soft Matter, 9:297-305, 2013.

[125] F Turci, C P Royall, and T Speck. Nonequilibrium phase transition in an atomistic glassformer: The connection to thermodynamics. Physical Review X, 7(3):031028, 2017.

[126] D Coslovich. Personal communication. 2017.

[127] Kenneth L. Kearns, Stephen F. Swallen, M D. Ediger, Tian Wu, and Lian Yu. Influence of substrate temperature on the stability of glasses prepared by vapor deposition. J. Chem. Phys., 127(15):154702, oct 2007.

[128] A Ninarello, L Berthier, and D Coslovich. Models and algorithms for the next generation of glass transition studies. Physical Review X, 7(2):021039, 2017.

[129] D. Cangialosi, A. Alegr?aa, and J. Colmenero. Effect of nanostructure on the thermal glass transition and physical aging in polymer materials. Prog. Poly. Sc., 54-?5:128?147, 2016.

[130] V. M. Boucher, D. Cangialosi, A. Alegr?aab, and J. Colmeneroab. Reaching the ideal glass transition by aging polymer films. Phys. Chem. Chem. Phys., 19:961-965, 2017.

[131] P. Luo, P. Wen, H. Y. Bai, B. Ruta, and W. H. Wang. Relaxation decoupling in metallic glasses at low temperatures. Phys. Rev. Lett.,, 118:225901, 2017.

[132] C. P. Royall, S. R. Williams, and H. Tanaka. Vitrification and gelation in sticky spheres. J. Chem. Phys., 148:044501, 2018.

[133] E Zaccarelli. Colloidal Gels: Equilibrium and non-equilibrium routes. J. Phys. Condens. Matter, 
19:323101, 2007.

[134] H. Tanaka. Viscoelastic phase seperation. J. Phys.: Condens. Matter, 12:R207-R264, 2000.

[135] V Testard, L Berthier, and W Kob. Influence of the glass transition on the liquid-gas spinodal decomposition. Phys. Rev. Lett., 106:125702, 2011.

[136] V. Testard, L. Bethier, and W. Kob. Intermittent dynamics and logarithmic domain growth during the spinodal decomposition of a glass-forming liquid. J. Chem. Phys., 140:164502, 2014.

[137] D. Cangialosi, V. M. Boucher, A. Alegría, and J. Colmenero. Direct evidence of two equilibration mechanisms in glassy polymers. Phys. Rev. Lett., 111:095701, 2013.

[138] D Huang, S L Simon, and G B McKenna. Equilibrium heat capacity of the glass-forming poly $(\alpha-$ methyl styrene) far below the kauzmann temperature: The case of the missing glass transition. J. Chem. Phys, 119:3590-3593, 2003.

[139] G B McKenna and J Zhao. Accumulating evidence for non-diverging time-scales in glass-forming fluids. J. Non-Cryst. Sol., 407:3-13, 2015.

[140] I Lyubimov, L Antony, D M. Walters, D Rodney, M D. Ediger, and J J. De Pablo. Orientational anisotropy in simulated vapor-deposited molecular glasses. J. Chem. Phys., 143:094502, 2015.

[141] S. S. Dalal, D. M. Walters, I. Lyubimov, J. J. de Pablo, and M. D. Ediger. Tunable molecular orientation and elevated thermal stability of vapor-deposited organic semiconductors. Proc. Nat. Acad. Sci., 112:4227?4232, 2015.

[142] S S Dalal and M D Fakhraai, Zand Ediger. High-Throughput Ellipsometric Characterization of Vapor-Deposited Indomethacin Glasses. J. Phys. ..., 2013.

[143] J Ràfols-Ribé, Gonzalez-Silveira. M., C Rodríguez-Tinico, and J Rodríguez-Viejo. The role of thermodynamic stability in the characteristics of the devitrification front of vapour-deposited glasses of toluene. Phys. Chem. Chem. Phys., 19:11089, 2017.

[144] J D. Stevenson and P Wolynes. On the surface of glasses. J. Chem. Phys., 129:234514, 2008.

[145] Y Zhang and Z Fakhraai. Decoupling of surface diffusion and relaxation dynamics of molecular glasses. Proc. Nat. Acad. Sci., 114:4915-?919, 2017.

[146] Y Zhang and Z Fakhraai. Invariant fast diffusion on the surfaces of ultrastable and aged molecular glasses. Phys. Rev. Lett., 118:066101, 2017.

[147] E A. A. Pogna, C Rodr?guez-Tinoco, G Cerullo, C Ferrante, J Rodr?guez-Viejo, and Tullio Scopigno. Probing equilibrium glass flow up to exapoise viscosities. Proc. Nat. Acad. Sci., 112:2331?2336, 2015.

[148] T Pérez-Castañeda, C Rodr?guez-Tinoco, J Rodr?guez-Viejo, and M A. Ramos. Suppression of tunneling two-level systems in ultrastable glasses of indomethacin. Proc. Nat. Acad. Sci., 111:11275, 2014.

[149] T Pérez-Castañeda, R J. Jiménez-Riobóo, and M A. Ramos. Two-level systems and boson peak remain stable in 110-million-year-old amber glass. Phys. Rev. Lett., 112:165901, 2014.

[150] C. A. Angell, Y. Yue, L.-M. Wang, J. R. D. Copley, S. Borick, and S. Mossa. Potential energy, relaxation, vibrational dynamics and the boson peak, of hyperquenched glasses. J. Phys.: Condens. Matter, 15:S1051-S1068, 2003.

[151] A Ivlev, H Löwen, G E Morfill, and C P Royall. Complex Plasmas and Colloidal Dispersions: Particle-resolved Studies of Classical Liquids and Solids. World Scientific Publishing Co., Singapore Scientific, 2012.

[152] G L Hunter and E R Weeks. The physics of the colloidal glass transition. Rep. Prog. Phys., 75:66501, 2012.

[153] U Gasser. Crystallization in three- and two-dimensional colloidal suspensions. J. Phys. Condens. Matter, 21:203101, 2009.

[154] J C Crocker and D G Grier. Methods of Digital Video Microscopy for Colloidal Studies. J. Coll. Interf. Sci., 179:298-310, 1995.

[155] C P Royall, M E Leunissen, and A van Blaaderen. A new colloidal model system to study long-range interactions quantitatively in real space. J. Phys. Condens. Matter, 15(48):S3581- 
The race to the bottom: approaching the ideal glass?

-S3596, dec 2003.

[156] A van Blaaderen and P Wiltzius. Real-space structure of colloidal hard-sphere glasses. Science (80-. )., 270(5239):1177-1179, nov 1995.

[157] E R Weeks, J C Crocker, A C Levitt, A Schofield, and D A Weitz. Three-dimensional direct imaging of structural relaxation near the colloidal glass transition. Science (80-. )., 287:627631, 2001.

[158] W K Kegel and A van Blaaderen. Direct observation of dynamic heterogeneities in colloidal hard-sphere suspensions. Science (80-. )., 287:290-293, 2001.

[159] D G. Grier. A revolution in optical manipulation. Nature, 424:810-816, 2003.

[160] S Gokhale, A K. Sood, and R Ganapathy. Deconstructing the glass transition through critical experiments on colloids. Adv. Phys., 65(4):363-452, 2016.

[161] S Gokhale, K H. Nagamanasa, A K. Sood, and R Ganapathy. Influence of an amorphous wall on the distribution of localized excitations in a colloidal glass-forming liquid. J. Stat. Mech.: Theory and Experiment, page 074013, 2016.

[162] I Williams, E C. Oğuz, P Bartlett, H Lowen, and C P. Royall. Flexible confinement leads to multiple relaxation regimes in glassy colloidal liquids. J. Chem. Phys., 142:024505, 2015.

[163] C P Royall, S R Williams, and Tanaka H. The nature of the glass and gel transitions in sticky spheres. arXiv:, page 1409.5469, 2014.

[164] G Brambilla, D El Masri, M Pierno, L Berthier, L Cipelletti, G Petekidis, and A B. Schofield. Probing the Equilibrium Dynamics of Colloidal Hard Spheres above the Mode-Coupling Glass Transition. Phys. Rev. Lett., 102(8):85703, 2009.

[165] S Gokhale, K H. Nagamanasa, R Ganapathy, and A K. Sood. Growing dynamical facilitation on approaching the random pinning colloidal glass transition. Nature Comm., 5:4685, 2014.

[166] I. Williams, F. Turci, J. E. Hallett, P. Crowther, C. Cammarota, G. Biroli, and Royall C. P. Experimental determination of configurational entropy in a two-dimensional liquid under random pinning. J. Phys.: Condens. Matter, 30:094003, 2018.

[167] X Cao, H Zhang, and Y Han. Release of free-volume bubbles by cooperative-rearrangement regions during the deposition growth of a colloidal glass. Nature Comm., 18:362, 2017.

[168] T Speck, A Malins, and C P Royall. First-Order Phase Transition in a Model Glass Former: Coupling of Local Structure and Dynamics. Phys. Rev. Lett., 109:195703, 2012.

[169] R. Pinchaipat, M. Campo, F. Turci, J. Hallett, T Speck, and C. P. Royall. Experimental evidence for a structural-dynamical transition in trajectory space. Phys. Rev. Lett., 119:028004, 2017.

[170] C Patrick Royall and Stephen R. Williams. The role of local structure in dynamical arrest. Phys. Rep., 560:1, 2015.

[171] Nicholas Bailey, Trond Ingebrigtsen, Jesper Schmidt Hansen, Arno Veldhorst, Lasse Bøhling, Claire Lemarchand, Andreas Olsen, Andreas Bacher, Lorenzo Costigliola, Ulf Pedersen, et al. Rumd: A general purpose molecular dynamics package optimized to utilize gpu hardware down to a few thousand particles. SciPost Physics, 3(6):038, 2017.

[172] Steve Plimpton. Fast parallel algorithms for short-range molecular dynamics. Journal of computational physics, 117(1):1-19, 1995.

[173] W Michael Brown, Peng Wang, Steven J Plimpton, and Arnold N Tharrington. Implementing molecular dynamics on hybrid high performance computers-short range forces. Computer Physics Communications, 182(4):898-911, 2011.

[174] Joshua A Anderson, Chris D Lorenz, and Alex Travesset. General purpose molecular dynamics simulations fully implemented on graphics processing units. Journal of Computational Physics, 227(10):5342-5359, 2008.

[175] Jens Glaser, Trung Dac Nguyen, Joshua A Anderson, Pak Lui, Filippo Spiga, Jaime A Millan, David C Morse, and Sharon C Glotzer. Strong scaling of general-purpose molecular dynamics simulations on gpus. Computer Physics Communications, 192:97-107, 2015.

[176] F H Stillinger and T A Weber. Dynamics of structural transitions in liquids. Phys. Rev. A, 28:2408-2416, 1983. 
[177] F H Stillinger and T A Weber. Packing structures and transitions in liquids and solids. Science (80-. )., 255:983-989, 1984.

[178] S Karmakara, C Dasguptaa, S Sastry, S Karmakar, C Dasgupta, and S Sastry. Growing length and time scales in glass-forming liquids. Proc. Nat. Acad. Sci. U.S.A., 106(10):3675-3679, 2009.

[179] S Sastry. The relationship between fragility, configurational entropy and the potential energy landscape of glass-forming liquids. Nature, 409:300-301, 2001.

[180] S Sastry, P G Debenedetti, and F H Stillinger. Signatures of distinct dynamical regimes in the energy landscape of a glass-forming liquid. Nature, 393:594-597, 1998.

[181] T B Schroder, S Sastry, J C Dyre, and S Glotzer. Crossover to potential energy landscape dominated dynamics in a model glass- forming liquid. J. Chem. Phys., 112:9834-9840, 2000.

[182] B Doliwa and A Heuer. Energy barriers and activated dynamics in a supercooled Lennard-Jones liquid. Phys. Rev. E, 67:31506, 2003.

[183] G A Appignanesi, J A Rodriguez Fris, R A Montani, and W Kob. Democratic Particle Motion for Metabasin Transitions in Simple Glass Formers. Phys. Rev. Lett., 96:57801, 2006.

[184] C Donati, J F Douglas, W Kob, S J Plimpton, P H Poole, and S C Glotzer. Stringlike Cooperative Motion in a Supercooled Liquid. Phys. Rev. Lett., 81:2338-2341, 1998.

[185] D J Wales. Energy Landscapes: Applications to Clusters, Biomolecules and Glasses. Cambridge University Press, Cambridge, 2004.

[186] F Calvo, T V Bogdan, V K De Souza, and D J Wales. Equilibrium density of states and thermodynamic properties of a model glass former. J. Chem. Phys., 127:44508, 2007.

[187] V K De Souza and D J Wales. Energy landscapes for diffusion: Analysis of cage-breaking processes. J. Chem. Phys., 129:164507, 2008.

[188] T F Middleton, J Hernandez-Rojas, P N Mortenson, and D J Wales. Crystals of binary LennardJones solids. Phys. Rev. B., 64:184201, 2001.

[189] D Coslovich and G Pastore. Understanding fragility in supercooled Lennard-Jones mixtures. I. Locally preferred structures. J. Chem. Phys, 127:124504, 2007.

[190] E Flenner and G Szamel. Fundamental differences between glassy dynamics in two and three dimensions. Nature communications, 6, 2015.

[191] D Frenkel and B Smit. Understanding Molecular Simulation: from Algorithms to Applications. New York: Academic, 2001.

[192] Robert H Swendsen and Jian-Sheng Wang. Replica monte carlo simulation of spin-glasses. Physical review letters, 57(21):2607, 1986.

[193] Koji Hukushima and Koji Nemoto. Exchange monte carlo method and application to spin glass simulations. Journal of the Physical Society of Japan, 65(6):1604-1608, 1996.

[194] Yuji Sugita and Yuko Okamoto. Replica-exchange molecular dynamics method for protein folding. Chemical physics letters, 314(1-2):141-151, 1999.

[195] David J Earl and Michael W Deem. Parallel tempering: Theory, applications, and new perspectives. Physical Chemistry Chemical Physics, 7(23):3910-3916, 2005.

[196] Shankar Kumar, John M Rosenberg, Djamal Bouzida, Robert H Swendsen, and Peter A Kollman. The weighted histogram analysis method for free-energy calculations on biomolecules. i. the method. Journal of computational chemistry, 13(8):1011-1021, 1992.

[197] Christian Bartels. Analyzing biased monte carlo and molecular dynamics simulations. Chemical Physics Letters, 331(5-6):446-454, 2000.

[198] E P Bernard and W Krauth. Two-Step Melting in Two Dimensions: First-Order Liquid-Hexatic Transition. Phys. Rev. Lett., 107(15):155704, oct 2011.

[199] E P Bernard, W Krauth, and D B Wilson. Event-chain Monte Carlo algorithms for hard-sphere systems. Phys. Rev. E, 80(5):56704, 2009.

[200] D Gazzillo and G Pastore. Equation of state for symmetric non-additive hard-sphere fluids: An approximate analytic expression and new Monte Carlo results. Chem. Phys. Lett., 159(4):388392, 1989. 
[201] T S Grigera and G Parisi. Fast Monte Carlo algorithm for supercooled soft spheres. Phys. Rev. E, 63(4):45102, 2001.

[202] L. Berthier, P. Charbonneau, A. Ninarello, M. Ozawa, and S. Yaida. Zero-temperature glass transition in two dimensions. ArXiV, page 1805.09035, 2018.

[203] M Wyart and M E Cates. Does a growing static length scale control the glass transition? arXiv preprint arXiv:1705.06588, 2017.

[204] L. Berthier, G. Biroli, Bouchaud J.-P., and G. Tarjus. Can the glass transition be explained without a growing static length scale? ArXiV, page 1805.12378, 2018.

[205] G. Szamel. A theory for the dynamics of glassy mixtures with particle size swaps. ArXiV, page $1805.02753,2018$.

[206] H. Ikeda, F. Zamponi, and A. Ikeda. Mean field theory of the swap monte carlo algorithm. ArXiV, page 1709.08557, 2017.

[207] C. Brito, E. Lerner, and M. Wyart. Theory for swap acceleration near the glass and jamming transitions. ArXiV, page 1801.03796, 2018.

[208] L. Berthier, P. Charbonneau, E. Flenner, and F. Zamponi. Origin of ultrastability in vapordeposited glasses. Phys. Rev. Lett., 119:188002, 2017.

[209] Y. Jin and H. Yoshino. Exploring the complex free-energy landscape of the simplest glass by rheology. Nature Comm., 8:14935, 2017.

[210] A Jaster. Computer simulations of the two-dimensional melting transition using hard disks. Phys. Rev. E, 59(3):2594, 1999.

[211] J Russo and H Tanaka. Assessing the role of static length scales behind glassy dynamics in polydisperse hard disks. Proc. Natl. Acad. Sci., 112(22):6920-6924, 2015.

[212] J Russo and N B Wilding. Disappearance of the hexatic phase in a binary mixture of hard disks. Phys. Rev. Lett., 2017.

[213] P Diaconis, S Holmes, and R M Neal. Analysis of a nonreversible Markov chain sampler. Ann. Appl. Probab., pages 726-752, 2000.

[214] E P Bernard and W Krauth. Addendum to ?Event-chain Monte Carlo algorithms for hard-sphere systems?. Phys. Rev. E, 86(1):17701, 2012.

[215] M Michel, S C Kapfer, and W Krauth. Generalized event-chain Monte Carlo: Constructing rejection-free global-balance algorithms from infinitesimal steps. J. Chem. Phys., 140(5):54116, 2014.

[216] E A J F Peters and G de With. Rejection-free Monte Carlo sampling for general potentials. Phys. Rev. E, 85(2):26703, 2012.

[217] L Berthier, G Biroli, J-P Bouchaud, W Kob, K Miyazaki, and D R Reichman. Spontaneous and induced dynamic fluctuations in glass formers. i. general results and dependence on ensemble and dynamics. The Journal of chemical physics, 126(18):184503, 2007.

[218] L Berthier, G Biroli, J-P Bouchaud, W Kob, K Miyazaki, and David R Reichman. Spontaneous and induced dynamic correlations in glass formers. ii. model calculations and comparison to numerical simulations. The Journal of chemical physics, 126(18):184504, 2007.

[219] E Flenner, M Zhang, and G Szamel. Analysis of a growing dynamic length scale in a glass-forming binary hard-sphere mixture. Phys. Rev. E, 83:51501, 2011.

[220] S Franz and G Parisi. On non-linear susceptibility in supercooled liquids. Journal of Physics: Condensed Matter, 12(29):6335, 2000.

[221] Z Rotman and E Eisenberg. Direct measurements of the dynamical correlation length indicate its divergence at an athermal glass transition. Physical Review Letters, 105(22):1-4, 2010.

[222] P Scheidler, W Kob, and K Binder. The relaxation dynamics of a supercooled liquid confined by rough walls. The Journal of Physical Chemistry B, 108(21):6673-6686, 2004.

[223] F Turci, G Tarjus, and C P Royall. From glass formation to icosahedral ordering by curving three-dimensional space. Physical Review Letters, 118(21):215501, 2017.

[224] S Franz and G Semerjian. Dynamical heterogeneities in glasses, colloids, and granular media, chapter The length. Oxford Univ. Press, 2011. 
[225] A Cavagna, T S Grigera, and P Verrocchio. Mosaic multistate scenario versus one-state description of supercooled liquids. Physical review letters, 98(18):187801, 2007.

[226] G Biroli, J P. Bouchaud, A Cavagna, T S Grigera, and P Verrochio. Thermodynamic signature of growing amorphous order in glass-forming liquids. Nat. Phys., 4:771-775, 2008.

[227] C Cammarota, A Cavagna, G Gradenigo, T S. Grigera, and P Verrochio. Numerical determination of the exponents controlling the relationship between time, length, and temperature in glass-forming liquids. J. Chem. Phys., 131:194901, 2009.

[228] L Berthier and W Kob. Static point-to-set correlations in glass-forming liquids. Phys. Rev. E, 011102:2-6, 2012.

[229] S. Karmakar and G. Parisi. Random pinning glass model. Proc. Nat. Acad. Sci., 110:2752-2757, 2013.

[230] W Kob and L Berthier. Probing a liquid to glass transition in equilibrium. Phys. Rev. Lett., 110(24):245702, jan 2013.

[231] R L Jack, A J Dunleavy, and Royall C P. Information-theoretic measurements of coupling between structure and dynamics in glass-formers. Phys. Rev. Lett, 113:95703, 2014.

[232] C J Fullerton and R L Jack. Investigating amorphous order in stable glasses by random pinning. Physical review letters, 112(25):255701, 2014.

[233] F F Abraham and G M White. Computer Simulation of Vapor Deposition on Two-Dimensional Lattices. J. Appl. Phys., 41(4):1841-1849, 1970.

[234] M Schneider, A Rahman, and Ivan K Schuller. Role of relaxation in epitaxial growth: a moleculardynamics study. Phys. Rev. Lett., 55(6):604, 1985.

[235] C C Battaile and D J Srolovitz. Kinetic Monte Carlo simulation of chemical vapor deposition. Annu. Rev. Mater. Res., 32(1):297-319, 2002.

[236] C C Battaile, D J Srolovitz, and J E Butler. Morphologies of diamond films from atomic-scale simulations of chemical vapor deposition. Diam. Relat. Mater., 6(9):1198-1206, 1997.

[237] L Meng, Q Sun, J Wang, and F Ding. Molecular dynamics simulation of chemical vapor deposition graphene growth on Ni (111) surface. J. Phys. Chem. C, 116(10):6097-6102, 2012.

[238] W Qi and R K Bowles. Vapor Condensed and Supercooled Glassy Nanoclusters. ACS Nano, 10(3):3416-3423, mar 2016.

[239] S Singh and J J de Pablo. A molecular view of vapor deposited glasses. J. Chem. Phys., 134(19):194903, 2011.

[240] S Singh, M D Ediger, and J J de Pablo. Ultrastable glasses from in silico vapour deposition. Nat. Mater., 12(2):139-144, jan 2013.

[241] S. Singh, M. D. Ediger, and J. J. de Pablo. Corrigendum: Ultrastable glasses from in silico vapour deposition. Nature Materials, 13:662, 2014.

[242] P H Lin, I Lyubimov, L Yu, M D. Ediger, and J J. De Pablo. Molecular modeling of vapordeposited polymer glasses. J. Chem. Phys., 140(20):204504, 2014.

[243] I Lyubimov, M D Ediger, and J J de Pablo. Model vapor-deposited glasses: growth front and composition effects. J Chem Phys., 139:144505, 2013.

[244] D R. Reid, I Lyubimov, M D. Ediger, and J J. de Pablo. Age and structure of a model vapourdeposited glass. Nature Comm., 7:13062, 2016.

[245] L Berthier, P Charbonneau, E Flenner, and Z Zamponi. How to create equilibrium vapordeposited glasses. arXiv preprint arXiv:1706.02738, 2017.

[246] L Zhu, CW Brian, SF Swallen, PT Straus, MD Ediger, and L Yu. Surface self-diffusion of an organic glass. Physical Review Letters, 106(25):256103, 2011.

[247] T Bodineau and B Derrida. Current fluctuations in nonequilibrium diffusive systems: an additivity principle. Phys. Rev. Lett., 92(18):180601, 2004.

[248] Y S Elmatad, R L Jack, D Chandler, and J P Garrahan. Finite-temperature critical point of a glass transition. Proc. Nat. Acad. Sci., 107:12793-12798, 2010.

[249] B Derrida, J L Lebowitz, and E R Speer. Free energy functional for nonequilibrium systems: an exactly solvable case. Phys. Rev. Lett., 87(15):150601, 2001. 
[250] H Touchette. The large deviation approach to statistical mechanics. Phys. Rep., 478(1):1-69, 2009.

[251] J P Garrahan, R L Jack, E Lecomte V. amd Pitard, K van Duijvendijk, and F van Wijland. Dynamical First-Order Phase Transition in Kinetically Constrained Models of Glasses. Phy. Rev. Lett., 98:195702, 2007.

[252] L O Hedges, R L Jack, J P Garrahan, and D Chandler. Dynamic Order-Disorder in Atomistic Models of Structural Glass Formers. Science (80-. )., 323:1309-1313, 2009.

[253] M Merolle, J P Garrahan, and D Chandler. Space-time thermodynamics of the glass transition. Proc. Natl. Acad. Sci. U. S. A., 102(31):10837-10840, 2005.

[254] T Speck and D Chandler. Constrained dynamics of localized excitations causes a non-equilibrium phase transition in an atomistic model of glass formers. J. Chem. Phys., 136:184509, 2012.

[255] F Turci and E Pitard. Large deviations and heterogeneities in a driven kinetically constrained model. EuroPhys. Lett., 94:10003, 2011.

[256] D Coslovich and R L Jack. Structure of inactive states of a binary Lennard-Jones mixture. J. Stat. Mech. Theory Exp., 2016(7):74012, 2016.

[257] F Ritort and P Sollich. Glassy dynamics of kinetically constrained models. Adv. Phys., 52(4):219342, 2003.

[258] P G Bolhuis, D Chandler, C Dellago, and P L Geissler. Transition path sampling: Throwing ropes over rough mountain passes, in the dark. Annu. Rev. Phys. Chem., 53(1):291-318, 2002.

[259] C Giardina, J Kurchan, and L Peliti. Direct evaluation of large-deviation functions. Phys. Rev. Lett., 96(12):120603, 2006.

[260] F. Turci, T. Speck, and C. P. Royall. Structural-dynamical transition in the wahnström mixture. accepted in Eur. Phys. J. E, 2018.

[261] M Ozawa, W Kob, A Ikeda, and K Miyazaki. Equilibrium phase diagram of a randomly pinned glass-former. Proc. Nat. Acad. Sci., 112:6914-6919, 2015.

[262] K Ito, C T Moynihan, and C A Angell. Thermodynamic determination of fragility in liquids and a fragile-to-strong liquid transition in water. Nature, 398:492-495, 1999.

[263] C Zhang, L Hu, Y Yue, and J C. Mauro. Fragile-to-strong transition in metallic glass-forming liquids. J. Chem. Phys., 133(1):14508, 2010. 